\title{
HOMOTHETY CURVATURE HOMOGENEITY AND HOMOTHETY HOMOGENEITY
}

\author{
E. GARCÍA-RÍO, P. GILKEY AND S. NIKČEVIĆ
}

\begin{abstract}
We examine the difference between several notions of curvature homogeneity and show that the notions introduced by Kowalski and Vanžurová are genuine generalizations of the ordinary notion of $k$-curvature homogeneity. The homothety group plays an essential role in the analysis. We give a complete classification of homothety homogeneous manifolds which are not homogeneous and which are not VSI and show that such manifolds are cohomogeneity one. We also give a complete description of the local geometry if the homothety character defines a split extension.
\end{abstract}

\section{INTRODUCTION}

Let $\mathcal{M}=(M, g)$ be a pseudo-Riemannian manifold of dimension $m \geq 2$. Let $\nabla^{k} R \in \otimes^{k+4} T^{*} M$ denote the $k^{\text {th }}$ covariant derivative of the curvature tensor and let $\nabla^{k} \mathfrak{R} \in \otimes^{k+2} T^{*} M \otimes \operatorname{End}(T M)$ denote the $k^{\text {th }}$ covariant derivative of the curvature operator. These are related by the identity:

$$
\nabla^{k} R\left(x_{1}, x_{2}, x_{3}, x_{4} ; x_{5}, \ldots, x_{k+4}\right)=g\left(\nabla^{k} \mathfrak{R}\left(x_{1}, x_{2} ; x_{5}, \ldots, x_{k+4}\right) x_{3}, x_{4}\right) .
$$

Definition 1.1. $\mathcal{M}$ is said to be $k$-curvature homogeneous if given $P, Q \in M$, there is a linear isometry $\phi: T_{P} M \rightarrow T_{Q} M$ so that $\phi^{*}\left(\nabla^{\ell} R_{Q}\right)=\nabla^{\ell} R_{P}$ for $0 \leq \ell \leq k$.

There is a slightly different version of curvature homogeneity that we shall discuss here and which, motivated by the seminal work of Kowalski and Vanžurová [10, 11, we shall call homothety $k$-curvature homogeneity. In Definition 1.1, we may replace the curvature tensor $R$ by the curvature operator $\mathfrak{R}$ since we are dealing with isometries. This is not the case when we deal with homotheties and the variance is crucial. We will establish the following result in Section 2,

Lemma 1.2. The following conditions are equivalent and if any is satisfied, then $\mathcal{M}$ will be said to be homothety $k$-curvature homogeneous:

(1) Given any two points $P, Q \in M$, there is a linear homothety $\Phi=\Phi_{P, Q}$ from $T_{P} M$ to $T_{Q} M$ so that if $0 \leq \ell \leq k$, then $\Phi^{*}\left(\nabla^{\ell} \mathfrak{R}_{Q}\right)=\nabla^{\ell} \mathfrak{R}_{P}$.

(2) Given any two points $P, Q \in M$, there exists a linear isometry $\phi=\phi_{P, Q}$ from $T_{P} M$ to $T_{Q} M$ and there exists $0 \neq \lambda=\lambda_{P, Q} \in \mathbb{R}$ so that if $0 \leq \ell \leq k$, then $\phi^{*}\left(\nabla^{\ell} R_{Q}\right)=\lambda^{-\ell-2} \nabla^{\ell} R_{P}$.

(3) There exist constants $\varepsilon_{i j}$ and constants $c_{i_{1} \ldots i_{\ell+4}}$ such that for all $Q \in M$, there is a basis $\left\{\xi_{1}^{Q}, \ldots, \xi_{m}^{Q}\right\}$ for $T_{Q} M$ and there exists $0 \neq \lambda_{Q} \in \mathbb{R}$ so that if $0 \leq \ell \leq k$, then for all $i_{1}, i_{2}, \ldots, g_{Q}\left(\xi_{i_{1}}^{Q}, \xi_{i_{2}}^{Q}\right)=\varepsilon_{i_{1} i_{2}}$ and

$$
\nabla^{\ell} R_{Q}\left(\xi_{i_{1}}^{Q}, \xi_{i_{2}}^{Q}, \xi_{i_{3}}^{Q}, \xi_{i_{4}}^{Q} ; \xi_{i_{5}}^{Q}, \ldots, \xi_{\ell+4}^{Q}\right)=\lambda_{Q}^{-\ell-2} c_{i_{1} \ldots i_{\ell+4}} .
$$

Remark 1.3. This agrees with Proposition 0.1 of Kowalski and Vanžurová [1]. If we can take $\lambda_{P, Q}=1$ for all $P$ and $Q$, then $\mathcal{M}$ is $k$-curvature homogeneous. But we shall see in Theorem 1.16, there are examples which are homothety 2-curvature

2000 Mathematics Subject Classification. 53C50, 53C44.

Key words and phrases. curvature homogeneity, homothety, cohomogeneity one. 
homogeneous which are not 2-curvature homogeneous and thus $\lambda$ varies with the point.

Motivated by Lemma 1.2, we make the following:

Definition 1.4. A $k$-curvature model is a tuple $\mathfrak{M}_{k}:=\left(V,\langle\cdot, \cdot\rangle, A^{0}, \ldots, A^{k}\right)$ where $\langle\cdot, \cdot\rangle$ is a non-degenerate inner product on an $m$-dimensional real vector space $V$ and where $A^{i} \in \otimes^{4+i}\left(V^{*}\right)$ satisfies the algebraic identities of $\nabla^{i} R$. We say that two $k$-curvature models $\mathfrak{M}_{k}^{1}$ and $\mathfrak{M}_{k}^{2}$ are homothety isomorphic if there is a linear isometry $\phi$ from $\left(V^{1},\langle\cdot, \cdot\rangle^{1}\right)$ to $\left(V^{2},\langle\cdot, \cdot\rangle^{2}\right)$ and if there exists $\lambda \in \mathbb{R}$ so that

$$
\phi^{*} A^{\ell, 2}=\lambda^{-\ell-2} A^{\ell, 1} \text { for all } 0 \leq \ell \leq k .
$$

Lemma 1.2 shows a pseudo-Riemannian manifold $\mathcal{M}$ is homothety $k$-curvature homogeneous if and only if there exists a $k$-curvature model $\mathfrak{M}_{k}$ so that $\mathfrak{M}_{k}$ is homothety isomorphic to $\left(T_{P} M, g_{P}, R_{P}, \ldots, \nabla^{k} R_{P}\right)$ for all $P$ in $M$.

1.1. Structure Groups. Let $\mathcal{D}(M)$ denote the group of diffeomorphisms of a pseudo-Riemannian manifold $\mathcal{M}$. We define the group of isometries $\mathcal{I}(\mathcal{M})$ and the group of homotheties $\mathcal{H}(\mathcal{M})$ by setting:

$$
\begin{aligned}
& \mathcal{I}(\mathcal{M}):=\left\{T \in \mathcal{D}(M): T^{*} g=g\right\}, \\
& \mathcal{H}(\mathcal{M}):=\left\{T \in \mathcal{D}(M): \exists \lambda(T)>0: T^{*} g=\lambda^{2} g\right\} .
\end{aligned}
$$

We say that $\mathcal{M}$ is homogeneous if $\mathcal{I}(\mathcal{M})$ acts transitively on $M$. Similarly, $\mathcal{M}$ is said to be homothety homogeneous if $\mathcal{H}(\mathcal{M})$ acts transitively on $M$. There are similar local notions where the transformation $T$ is not assumed globally defined.

A complete Riemannian manifold which admits a non-Killing homothety vector field must be flat [17, and hence it follows that a non-flat complete homothety homogeneous manifold is necessarily homogeneous in the Riemannian setting; we give another proof of this result based on the slices of Theorem 1.6. The situation is not so rigid in the Lorentzian case where pp-wave metrics support non-Killing homothety vector fields (see for example [2, 12, 16] and references therein).

1.2. Stability. Assertion (1) in the following result was established by Singer [15] in the Riemannian context and by Podesta and Spiro [14] in the pseudo-Riemannian setting. In Section 3, we will use results of [14] to establish Assertion (2) which extends these results to the homothety setting. Recall that the linear orthogonal group $\mathcal{O}$ and the linear homothety group $\mathcal{H O}$ in dimension $m$ but arbitrary signature satisfy:

$$
\operatorname{dim}\{\mathcal{O}\}:=\frac{1}{2} m(m-1) \text { and } \operatorname{dim}\{\mathcal{H O}\}=\frac{1}{2} m(m-1)+1 .
$$

Theorem 1.5. Let $\mathcal{M}=(M, g)$ be a pseudo-Riemannian manifold.

(1) The following Assertions are equivalent:

(a) $\mathcal{M}$ is locally homogeneous.

(b) $\mathcal{M}$ is $k$-curvature homogeneous for all $k$.

(c) $\mathcal{M}$ is $k$-curvature homogeneous for $k=\frac{1}{2} m(m-1)$.

(2) The following Assertions are equivalent:

(a) $\mathcal{M}$ is locally homothety homogeneous.

(b) $\mathcal{M}$ is homothety $k$-curvature homogeneous for all $k$.

(c) $\mathcal{M}$ is homothety $k$-curvature homogeneous for $k=\frac{1}{2} m(m-1)+1$.

1.3. Homothety homogeneous manifolds. Suppose that $\mathcal{M}=(M, g)$ is a connected homothety homogeneous pseudo-Riemannian manifold. Let $\mathcal{H}$ be a connected Lie subgroup of the group of homotheties of $\mathcal{M}$ which acts transitively on $\mathcal{M}$; note that $\mathcal{H}$ is not necessarily the full group of homotheties. If $\Phi \in \mathcal{H}$, let $\lambda(\Phi)>0$ be the homothety constant so that $\Phi^{*}(g)=\lambda(\Phi)^{2} g$. Then $\lambda$ is a 
smooth multiplicative character on $\mathcal{H}$ since $\lambda\left(\Phi_{1} \Phi_{2}\right)=\lambda\left(\Phi_{1}\right) \lambda\left(\Phi_{2}\right)$ for $\Phi_{i} \in \mathcal{H}$. Let $\mathcal{I}=\operatorname{ker}(\lambda)$ be the subgroup consisting of all the isometries which are contained in $\mathcal{H}$. We suppose $\mathcal{I} \neq \mathcal{H}$ or, equivalently, that $\lambda$ is non-trivial. Let $\mathcal{R}$ be a Weyl scalar invariant of order $\ell$ (i.e., it involves a total of $\ell$ derivatives of the metric tensor); such invariants are constructed from the covariant derivatives of the curvature tensor by contracting indices in pairs where we adopt the Einstein convention and sum over repeated indices. For example, the scalar curvature $\tau$, the norm of the curvature tensor $|R|^{2}$, the norm of the Ricci tensor $|\rho|^{2}$, and the Laplacian of the scalar curvature $\Delta \tau$ are Weyl scalar invariants of orders $2,4,4$, and 4 , respectively, that are defined by:

$$
\begin{array}{ll}
\tau:=g^{i l} g^{j k} R_{i j k l}, & |R|^{2}:=g^{i a} g^{j b} g^{k c} g^{l d} R_{i j k l} R_{a b c d}, \\
|\rho|^{2}:=g^{i a} g^{j b} g^{k c} g^{l d} R_{i j b l} R_{a k c d}, & \Delta \tau:=-g^{i a} g^{j b} g^{k c} R_{i j b a ; k c} .
\end{array}
$$

If $\mathcal{R}$ is a Weyl scalar invariant of order $\ell$, then the computations used to establish Lemma 1.2 show that:

$$
\Phi^{*}(\mathcal{R})=\lambda(\Phi)^{-\ell} \mathcal{R} \quad \text { for any } \quad \Phi \in \mathcal{H} .
$$

The manifold $\mathcal{M}$ is said to be a VSI (vanishing scalar invariants) manifold if all the Weyl scalar invariants vanish identically; we refer to [1, 3, 4, 5] for further details. In the Riemannian setting, $\mathcal{M}$ is VSI if and only if $\mathcal{M}$ is flat. There are non-flat VSI manifolds in the higher signature setting which are homothety homogeneous as we shall discuss in Theorem 1.16. If $\mathcal{M}$ is not VSI, then $\mathcal{M}$ is not homogeneous since some Weyl scalar invariant is non-constant.

Let $\mathcal{R}$ be a Weyl scalar invariant of order $\ell$ which does not vanish identically at some point; by Equation (1.a), $\mathcal{R}$ never vanishes on $M$ since $\mathcal{H}$ acts transitively. In the Riemannian setting, we may take $\mathcal{R}=|R|^{2}$. If $P_{0}$ is the base point of $M$, set:

$$
\mu_{\mathcal{R}}(P):=\left|\frac{\mathcal{R}\left(P_{0}\right)}{\mathcal{R}(P)}\right|^{1 / \ell} .
$$

We will establish the following Theorem in Section 4 which shows that $\mathcal{M}$ has cohomogeneity 1 :

Theorem 1.6. Let $\mathcal{M}=(M, g)$ be a connected pseudo-Riemannian manifold which is homothety homogeneous with non-trivial homothety character $\lambda$ which is not VSI. Let $\mathcal{R}$ be a scalar Weyl invariant which does not vanish on $M$. Then

(1) The level sets $M_{c}^{\mathcal{R}}=\left\{P \in M: \mu_{\mathcal{R}}(P)=c\right\}$ are smooth submanifolds of $M$ which have codimension 1 , which are independent of the particular $\mathcal{R}$ chosen, and on which $\mathcal{I}$ acts transitively.

(2) If $\mathcal{M}$ is Riemannian, then there exists a constant $\kappa=\kappa(M)>0$ so that $\operatorname{dist}\left(M_{c}^{\mathcal{R}}, M_{d}^{\mathcal{R}}\right)=\kappa|c-d|$. Furthermore, $\mathcal{M}$ is incomplete.

In order to describe the local structure of homothety homogeneous manifolds which are not VSI, we make the following:

Definition 1.7. A quadruple $\mathcal{P}=\left(M, g_{0}, \mathcal{H}, \lambda\right)$ is said to be an $\mathfrak{P}$-structure if:

(1) $\mathcal{H}$ is a connected Lie group acting smoothly, transitively, and effectively by isometries on the connected pseudo-Riemannian manifold $\mathcal{M}_{0}=\left(M, g_{0}\right)$.

(2) $\lambda$ is a non-trivial, smooth $\mathbb{R}^{+}$valued multiplicative character of $\mathcal{H}$.

(3) Let $P_{0}$ be the base point of $M$ and let $\mathcal{H}_{0}:=\left\{\Phi \in \mathcal{H}: \Phi P_{0}=P_{0}\right\}$ be the isotropy subgroup of the action. Then $\mathcal{H}_{0} \subset \operatorname{ker}(\lambda)$.

If $\mathcal{P}=\left(M, g_{0}, \mathcal{H}, \lambda\right)$ is a $\mathfrak{P}$ structure, let $M_{t}:=\lambda^{-1}\{t\} / \mathcal{H}_{0}$. Since $\operatorname{ker}(\lambda)$ acts transitively by isometries on each $M_{t}, \mathcal{M}_{t}:=\left(M_{t},\left.g_{0}\right|_{M_{t}}\right)$ is a homogeneous submanifold of $M$ which has codimension 1. If $P \in M_{t}$, let $g_{\lambda}(P)=t^{2} g_{0}(P)$ define a metric which is conformally equivalent to $g_{0}$. Let $\mathcal{M}_{\mathfrak{P}}:=\left(M, g_{\lambda}\right)$ be the associated 
pseudo-Riemannian manifold. We will establish the following result in Section 5 classifying homothety homogeneous manifolds with non-trivial homothety character which are not VSI; the classification is still open in the VSI context:

\section{Theorem 1.8.}

(1) If $\mathcal{P}$ is a $\mathfrak{P}$ structure, then $\mathcal{M}_{\mathfrak{P}}$ is a homothety homogeneous manifold with homothety character $\lambda$.

(2) If $\mathcal{M}=(M, g)$ is a homothety homogeneous manifold with non-trivial homothety character which is not VSI, then $M=\mathcal{M}_{\mathcal{P}}$ for some $\mathfrak{P}$ structure.

Let $\mathcal{M}=(M, g)$ be a connected homothety homogeneous pseudo-Riemannian manifold and let $\mathcal{H}$ be a Lie subgroup of homotheties acting transitively on $\mathcal{M}$. We suppose that $\mathcal{M}$ is not VSI and that the homothety multiplicative character $\lambda$ is non-trivial. Let $\mathcal{L}$ be the Lie derivative. Recall that a vector field $X$ is said to be a Killing vector field if $\mathcal{L}_{X} g=0$ or, equivalently, if $X_{a ; b}+X_{b ; a}=0$ where ';' represents covariant differentiation with respect to the Levi-Civita connection. Similarly $X$ is said to be a homothety vector field if $\mathcal{L}_{X} g=\lambda g$ or, equivalently, $X_{a ; b}+X_{b ; a}=2 \lambda g_{a b}$. Let $\mathfrak{H}$ be the Lie algebra of $\mathcal{H}$ and let $\mathfrak{I}$ the Lie sub algebra of $\mathcal{I}$; the elements of $\mathfrak{H}$ are homothety vector fields and the elements of $\mathfrak{I}$ are Killing vector fields. The short exact sequence

$$
1 \rightarrow \operatorname{ker}(\lambda) \rightarrow \mathcal{H} \stackrel{\lambda}{\rightarrow} \mathbb{R}^{+} \rightarrow 1
$$

defined by the homothety character is crucial. This sequence is said to split if $\mathcal{H}$ is isomorphic as a Lie group to $\mathbb{R}^{+} \oplus \operatorname{ker}(\lambda)$ and if under this isomorphism, $\lambda$ is projection on the first factor. The sequence is split if and only if there exists $0 \neq \xi \in \mathfrak{H}$ so that $[\xi, \eta]=0$ for all $\eta \in \mathfrak{H}$; such a $\xi$ generates a 1 -parameter subgroup $\Phi_{t}$ such that $\lambda\left(\Phi_{t}\right)=t$ for $t \in \mathbb{R}^{+}$that is central in $\mathcal{H}$ and gives rise to the splitting. In the Riemannian setting, if $\mathcal{I}$ is compact (or, equivalently, a slice $M_{c}$ is compact), then we can average over the group to construct such an element of $\mathfrak{H}$ and show the sequence is necessarily split in this setting.

In view of Theorem 1.8, it is perhaps worth to exhibit a Lie group with many multiplicative $\mathbb{R}^{+}$valued characters; there, are of course, many possible such examples:

Example 1.9. Let $\mathcal{H}_{n}$ be the group of upper triangular $n \times n$ real matrices with positive entries on the diagonal. A typical element $H \in \mathcal{H}$ takes the form:

$$
H:=\left(\begin{array}{lllll}
h_{11} & h_{12} & h_{13} & \ldots & h_{1 n} \\
0 & h_{22} & h_{23} & \ldots & h_{2 n} \\
0 & 0 & h_{33} & \ldots & h_{3 n} \\
\ldots & \ldots & \ldots & \ldots & \ldots \\
0 & 0 & 0 & \ldots & h_{n n}
\end{array}\right)
$$

where $h_{11}>0, \ldots, h_{n n}>0$, and where the $h_{i j}$ are arbitrary real numbers for $i<j$. If $0 \neq \vec{a}=\left(a_{1}, \ldots, a_{n}\right) \in \mathbb{R}^{n}$, then we may define a multiplicative $\mathbb{R}^{+}$valued character $\lambda_{\vec{a}}$ on $\mathcal{H}$ by setting

$$
\lambda_{\vec{a}}(H)=h_{11}^{a_{1}} \ldots h_{n n}^{a_{n}} .
$$

The associated sequence $1 \rightarrow \operatorname{ker}\left(\lambda_{\vec{a}}\right) \rightarrow \mathcal{H}_{n} \rightarrow \mathbb{R}^{+} \rightarrow 1$ is split if and only if $a_{1}+\cdots+a_{n} \neq 0$. Thus there are non-split characters. If we were to permit the $h_{i j}$ to be complex with $h_{i i} \neq 0$, we could define $\mathcal{H}_{\mathbb{C}}$ and obtain similarly

$$
\lambda_{\vec{a}}(H)=\left|h_{11}\right|^{a_{1}} \ldots\left|h_{n n}\right|^{a_{n}} .
$$

Definition 1.10. A quadruple $\mathcal{Q}=\left(N, g_{N}, \mathcal{I}, \theta\right)$ is said to be a $\mathfrak{Q}$-structure if $\mathcal{I}$ is a connected Lie group which acts transitively and effectively by isometries on a 
connected pseudo-Riemannian manifold $\left(N, g_{N}\right)$, and if $\theta \in C^{\infty}\left(\Lambda^{1} N\right)$ is invariant under the action of $\mathcal{I}$. Let $t \in \mathbb{R}$, let $M=\mathbb{R} \times N$, and let

$$
\mathcal{M}_{t, \mathcal{Q}}:=\left(\mathbb{R} \times N, g_{t, \mathcal{Q}}\right) \text { where } g_{t, \mathcal{Q}}:=e^{t x}\left(d x^{2}+d x \circ \theta+d s_{N}^{2}\right) .
$$

We say that $\mathfrak{Q}$ is a Riemannian $\mathfrak{Q}$ structure if $\mathcal{M}_{t, \mathcal{Q}}$ is Riemannian or, equivalently, if $\left(N, g_{N}\right)$ is Riemannian and if $\|\theta\|_{g_{N}}<1$.

We will establish the following result in Section 6 .

\section{Theorem 1.11.}

(1) Let $\mathcal{Q}=\left(N, g_{N}, \mathcal{I}, \theta\right)$ be a $\mathfrak{Q}$-structure. Then $g_{t, \mathcal{Q}}$ is non-degenerate if and only if $g_{N}(\theta, \theta) \neq 1$. In this setting, $\mathcal{M}_{t, \mathcal{Q}}$ is homothety homogeneous with non-trivial homothety character $\lambda$ and the short exact sequence defined by $\lambda$ splits.

(2) Let $\mathcal{M}$ be a homothety homogeneous Riemannian manifold with non-trivial homothety character $\lambda$ which is not VSI. If the short exact sequence defined by $\lambda$ splits and if $\xi$ is spacelike, then $\mathcal{M}=\mathcal{M}_{t, \mathcal{Q}}$ for some $\mathfrak{Q}$-structure and for some $t$ where $g_{N}(\theta, \theta) \neq 1$.

Theorem 1.12. If $\mathcal{Q}=\left(N, g_{N}, \mathcal{I}, \theta\right)$ is a Riemannian $\mathfrak{Q}$-structure, then $\mathcal{M}_{t, \mathcal{Q}}$ is isometric to $\mathcal{M}_{\tilde{t}, \tilde{\mathcal{Q}}}$ where $\tilde{\mathcal{Q}}=\left(\tilde{N}, \tilde{g}_{N}, \tilde{\mathcal{I}}, 0\right)$ has associated 1 -form $\tilde{\theta}=0$ if and only if $\theta_{a ; b}+\theta_{b ; a}=0$ for all $(a, b)$.

Of particular interest is the case when $\theta=0$. We shall prove the following result in Section 8

Theorem 1.13. Let $\mathcal{Q}=\left(N, g_{N}, \mathcal{I}, \theta\right)$ be a $\mathfrak{Q}$-structure with $\theta=0$. If $t \neq 0$, and if $\tau_{N}-\frac{(m-1)(m-2)}{4} t^{2} \neq 0$, then $\mathcal{M}$ is not flat. Such manifolds provide examples of manifolds which are $k$-homothety curvature homogeneous for all $k$ but which is not 0-curvature homogeneous.

1.4. Walker Lorentzian 3 dimensional manifolds. Section 9 is devoted to the study of a very specific family of examples. Let $\mathcal{M}=\left(M, g_{M}\right)$ be a 3 -dimensional Lorentzian manifold which admits a parallel null vector field, i.e. $\mathcal{M}$ is a 3 dimensional Walker manifold. Such a manifold admits local adapted coordinates $(x, y, \tilde{x})$ so that the (possibly) non-zero components of the metric are given by

$$
g\left(\partial_{x}, \partial_{x}\right)=-2 f(x, y), \quad g\left(\partial_{x}, \partial_{\tilde{x}}\right)=g\left(\partial_{y}, \partial_{y}\right)=1 .
$$

We clear the previous notation and denote this manifold by $\mathcal{M}_{f}$.

Walker manifolds are a special class of Kundt spacetimes, and thus they are Lorentzian manifolds with constant scalar curvature invariants [4, 5]. Moreover all the scalar curvature invariants of a Walker manifold vanish and we have:

Theorem 1.14. The manifolds $\mathcal{M}_{f}$ are all VSI manifolds.

Theorem 1.14 shows that these form an interesting family of examples. We have (see 8] Theorem 2.10 and Theorem 2.12):

Theorem 1.15. Suppose that $f_{y y}$ is never zero.

(1) $\mathcal{M}_{f}$ is 1-curvature homogeneous if and only if exactly one of the following three possibilities holds:

(a) $f_{y y}(x, y)=a y^{2}$ for $a \neq 0$. This manifold is symmetric.

(b) $f_{y y}(x, y)=\alpha(x) e^{b y}$ where $0 \neq b \in \mathbb{R}$ and where $\alpha(x)$ is arbitrary.

(c) $f_{y y}(x, y)=\alpha(x)$ where $\alpha(x)=c \cdot \alpha_{x}^{3 / 2}$ for some $c \neq 0 \in \mathbb{R}$.

(2) $\mathcal{M}$ is 2-curvature homogeneous if and only if it falls into one of the three families, all of which are locally homogeneous: 
(a) $f=b^{-2} \alpha(x) e^{b y}+\eta(x) y+\gamma(x)$ where $0 \neq b \in \mathbb{R}$, where $\alpha(x) \neq 0$, and where $\eta(x)=b^{-1} \alpha^{-1}(x)\left\{\alpha_{x x}(x)-\alpha_{x}^{2}(x) \alpha(x)^{-1}\right\}$.

(b) $f=a\left(x-x_{0}\right)^{-2} y^{2}+\beta(x) y+\gamma(x)$ where $0 \neq a \in \mathbb{R}$.

(c) $f=a y^{2}+\beta(x) y+\gamma(x)$ where $0 \neq a \in \mathbb{R}$.

We will establish the following analogue of Theorem 1.15 for homothety curvature homogeneity in Section 9 .

Theorem 1.16. Suppose $f_{y y}$ is never zero and non-constant.

(1) If $f_{y y y}$ never vanishes, then $\mathcal{M}_{f}$ is homothety 1-curvature homogeneous.

(2) If $f_{y y}=\alpha(x)$ with $\alpha_{x}$ never zero, then $\mathcal{M}_{f}$ is homothety 1-curvature homogeneous if and only if $f=a\left(x-x_{0}\right)^{-2} y^{2}+\beta(x) y+\gamma(x)$ where $0 \neq a \in \mathbb{R}$. This manifold is locally homogeneous.

(3) Assume that $\mathcal{M}_{f}$ is homothety 2-curvature homogeneous, and that $f_{y y}$ and $f_{\text {yyy }}$ never vanish. Then $\mathcal{M}_{f}$ is locally isometric to one of the examples:

(a) $\mathcal{M}_{ \pm e^{a y}}$ for some $a \neq 0$ and $M=\mathbb{R}^{3}$. This manifold is homogeneous.

(b) $\mathcal{M}_{ \pm \ln (y)}$ and $M=\mathbb{R} \times(0, \infty) \times \mathbb{R}$. This manifold is a homothety homogeneous manifold which is not locally homogeneous but which is cohomogeneity one.

(c) $\mathcal{M}_{ \pm y^{\varepsilon}}$ for $\varepsilon \neq 0,1,2$ and $M=\mathbb{R} \times(0, \infty) \times \mathbb{R}$. This manifold is a homothety homogeneous manifold which is not locally homogeneous but which is cohomogeneity one.

We also refer to recent work of Dunn and McDonald [7] for related work on homothety curvature homogeneous manifolds.

1.5. Variable homothety curvature homogeneity. In fact, the definition we have used in this paper differs subtly but in an important fashion from that originally given by Kowalski and Vanžurová 11; in that paper the scaling constant $\lambda$ was permitted to depend on $\ell$ and this gives rise to the notion of variable homothety $k$-curvature homogeneity. There are 4 different definitions which may be summarized as follows; we repeat two of the definitions to put the new definitions in context:

Definition 1.17. Let $\mathfrak{M}_{k}:=\left\{V,\langle\cdot, \cdot\rangle, A^{0}, \ldots, A^{k}\right\}$ be a $k$-curvature model and let $\mathcal{M}=(M, g)$ be a pseudo-Riemannian manifold.

(1) Static isometries that are independent of $k$. Recall that:

(a) $\mathcal{M}$ is $k$-curvature homogeneous with model $\mathfrak{M}_{k}$ if for any $P$ in $M$, there exists an isometry $\phi_{P}: T_{P} M \rightarrow V$ so $\phi_{P}^{*} A^{\ell}=\nabla^{\ell} R_{P}$ for $0 \leq \ell \leq k$.

(b) $\mathcal{M}$ is homothety $k$-curvature homogeneous with model $\mathfrak{M}_{k}$ if for any $P$ in $M$, there is an isometry $\phi_{P}: T_{P} M \rightarrow V$ and a scaling factor $0 \neq \lambda \in \mathbb{R}$ so that $\phi_{P}^{*} A^{\ell}=\lambda^{2+\ell} R_{P}$ for $0 \leq \ell \leq k$.

(2) Variable isometries that depend on $k$. We shall say that:

(a) $\mathcal{M}$ is variable- $k$-curvature homogeneous with model $\mathfrak{M}_{k}$ if for every $P$ in $M$ and if for every $0 \leq \ell \leq k$, there exist isometries $\phi_{P, \ell}: T_{P} M \rightarrow V$ so that $\phi_{P, \ell}^{*} A^{\ell}=\nabla^{\ell} R_{P}$.

(b) We say that $\mathcal{M}$ is variable homothety $k$-curvature homogeneous with model $\mathfrak{M}_{k}$ if for every $P$ in $M$ and if for every $0 \leq \ell \leq k$, there are linear isometries $\phi_{P, \ell}: T_{P} M \rightarrow V$ and scaling factors $0 \neq \lambda_{\ell} \in \mathbb{R}$ so that $\phi_{P, \ell}^{*} A^{\ell}=\lambda_{\ell}^{2+\ell} R_{P}$.

In Section 10, we will use the examples which were studied in Section 9 to show that Theorem [1.5 fails in the context of variable curvature homogeneity and hence also for variable homothety curvature homogeneity:

Theorem 1.18. 
(1) Let $k$ be a positive integer. There exists $f_{k}$ so that $\mathcal{M}_{f_{k}}$ is variable $k$ curvature homogeneous but not variable $k+1$-curvature homogeneous.

(2) The manifold $\mathcal{M}_{\frac{1}{2} e^{x} y^{2}}$ is variable $k$-curvature homogeneous for all $k$, but is not homothety 1-curvature homogeneous and hence not locally homogeneous.

(3) In Definition 1.17 we have the following implications:
$(1 a) \Rightarrow$
$(1 b) \Rightarrow$
(2b) and (1a) $\Rightarrow$
$(2 a) \Rightarrow$
$(2 b)$.

All other possible implications are false.

\section{The proof of Lemma 1.2}

Assume that Assertion (1) of Lemma 1.2 holds. This means that given any two points $P$ and $Q$ in $M$, there exists a linear homothety $\Phi: T_{P} M \rightarrow T_{Q} M$ so that if $0 \leq \ell \leq k$ and if $\left\{x_{i}\right\}$ are vectors in $T_{P} M$, then we have that:

$$
\begin{aligned}
& g_{Q}\left(\Phi x_{1}, \Phi x_{2}\right)=\lambda^{2} g_{P}\left(x_{1}, x_{2}\right), \text { and } \\
& \Phi\left\{\nabla^{\ell} \mathfrak{R}_{P}\left(x_{1}, x_{2} ; x_{5}, \ldots, x_{\ell+4}\right) x_{3}\right\}=\nabla^{\ell} \mathfrak{R}_{Q}\left(\Phi x_{1}, \Phi x_{2} ; \Phi x_{5}, \ldots, \Phi x_{\ell+4}\right) \Phi x_{3} .
\end{aligned}
$$

Taking the inner product with $\Phi x_{4}$ permits us to rewrite the second condition, which involves the curvature operator, in terms of the curvature tensor:

$$
\begin{aligned}
& \lambda^{2} \nabla^{\ell} R_{P}\left(x_{1}, x_{2}, x_{3}, x_{4} ; x_{5}, \ldots, x_{\ell+4}\right) \\
= & \lambda^{2} g_{P}\left(\nabla^{\ell} \mathfrak{R}_{P}\left(x_{1}, x_{2} ; x_{5}, \ldots, x_{\ell+4}\right) x_{3}, x_{4}\right) \\
= & g_{Q}\left(\Phi \nabla^{\ell} \mathfrak{R}_{P}\left(x_{1}, x_{2} ; x_{5}, \ldots, x_{\ell+4}\right) x_{3}, \Phi x_{4}\right) \\
= & g_{Q}\left(\nabla^{\ell} \mathfrak{R}_{Q}\left(\Phi x_{1}, \Phi x_{2} ; \Phi x_{5}, \ldots, \Phi x_{\ell+4}\right) \Phi x_{3}, \Phi x_{4}\right) \\
= & \nabla^{\ell} R_{Q}\left(\Phi x_{1}, \Phi x_{2}, \Phi x_{3}, \Phi x_{4} ; \Phi x_{5}, \ldots, \Phi x_{\ell+4}\right) .
\end{aligned}
$$

We set $\phi:=\lambda^{-1} \Phi$. We can rewrite these equations in the form:

$$
\begin{aligned}
& g_{Q}\left(\phi x_{1}, \phi x_{2}\right)=\lambda^{-2} g_{Q}\left(\Phi x_{1}, \Phi x_{2}\right)=g_{P}\left(x_{1}, x_{2}\right), \\
& \lambda^{2} \nabla^{\ell} R_{P}\left(x_{1}, x_{2}, x_{3}, x_{4} ; x_{5}, \ldots, x_{\ell+4}\right) \\
= & \nabla^{\ell} R_{Q}\left(\Phi x_{1}, \Phi x_{2}, \Phi x_{3}, \Phi x_{4} ; \Phi x_{5}, \ldots, \Phi x_{\ell+4}\right) \\
= & \lambda^{\ell+4} \nabla^{\ell} R_{Q}\left(\phi x_{1}, \phi x_{2}, \phi x_{3}, \phi x_{4} ; \phi x_{5}, \ldots, \phi x_{\ell+4}\right) \\
= & \lambda^{\ell+4} \phi^{*}\left(\nabla^{\ell} R_{Q}\right)\left(x_{1}, x_{2}, x_{3}, x_{4} ; x_{5}, \ldots, x_{\ell+4}\right) .
\end{aligned}
$$

This shows $\phi$ is an isometry from $T_{P} M$ to $T_{Q} M$ so $\phi^{*}\left(\nabla^{\ell} R_{Q}\right)=\lambda^{-2-\ell} \nabla^{\ell} R_{P}$. Consequently in Lemma 1.2. Assertion (1) $\Rightarrow$ Assertion (2); the proof of the converse implication is similar and will be omitted.

Suppose that Assertion (2) of Lemma 1.2 holds. Fix a basis $\left\{\xi_{1}^{P_{0}}, \ldots, \xi_{m}^{P_{0}}\right\}$ for $T_{P_{0}} M$ where $P_{0}$ is the base point of $M$. Set $c_{i_{1}, \ldots, i_{\ell+4}}:=\nabla^{\ell} R\left(\xi_{i_{1}}, \ldots, \xi_{i_{\ell+4}}\right)$ and set $\varepsilon_{i j}:=g_{P_{0}}\left(\xi_{i}, \xi_{j}\right)$. Let $Q \in M$. By assumption, there is an isometry $\phi$ from $T_{P_{0}} M$ to $T_{Q} M$ so:

$$
\phi^{*}\left(\nabla^{\ell} R_{Q}\right)=\lambda_{Q}^{-\ell-2} \nabla^{\ell} R_{P_{0}} \text { for } 0 \leq \ell \leq k .
$$

Set $\xi_{i}^{Q}:=\phi \xi_{i}^{P_{0}}$. Then:

$$
\begin{aligned}
& g_{Q}\left(\xi_{i}^{Q}, \xi_{j}^{Q}\right)=g_{Q}\left(\phi \xi_{i}^{P}, \phi \xi_{j}^{P}\right)=g_{P_{0}}\left(\xi_{i}^{P_{0}}, \xi_{j}^{P_{0}}\right)=\varepsilon_{i j} \\
& \nabla^{\ell} R_{Q}\left(\xi_{i_{1}}^{Q}, \ldots, \xi_{i_{\ell+4}}^{Q}\right)=\nabla^{\ell} R_{Q}\left(\phi \xi_{i_{1}}^{P_{0}}, \ldots, \phi \xi_{i_{\ell+4}}^{P_{0}}\right) \\
& \quad=\lambda_{Q}^{-\ell-2} \nabla^{\ell} R_{P_{0}}\left(\xi_{i_{1}}^{P_{0}}, \ldots, \xi_{i_{\ell+4}}^{P_{0}}\right)=\lambda_{Q}^{-\ell-2} c_{i_{1}, \ldots, i_{\ell+4}}
\end{aligned}
$$

This shows in Lemma 1.2 that Assertion (2) $\Rightarrow$ Assertion (3); the proof of the converse implication is similar and will be omitted. 


\section{The Proof of Theorem 1.5}

Fix the signature $(p, q)$ throughout this section where $m=p+q$. Let $(V,\langle\cdot, \cdot\rangle)$ be an inner product space of signature $(p, q)$. Let $\mathcal{O}$ and $\mathcal{H O}$ be the linear orthogonal group and linear homothety group of signature $(p, q)$ respectively;

$$
\begin{aligned}
& \mathcal{O}:=\left\{T \in \mathrm{GL}(V): T^{*}\langle\cdot, \cdot\rangle=\langle\cdot, \cdot\rangle\right\}, \\
& \left.\mathcal{H O}:=\left\{T \in \mathrm{GL}(V): T^{*}\langle\cdot, \cdot\rangle=\lambda^{2}\langle\cdot, \cdot\rangle\right\} \text { for some } 0<\lambda \in \mathbb{R}\right\} .
\end{aligned}
$$

Note that $\mathcal{H O}=\mathbb{R}^{+} \times \mathcal{O}$. Fix a basis $\left\{v_{1}, \ldots, v_{m}\right\}$ for $V$ and let $\epsilon_{i j}:=\left\langle v_{i}, v_{j}\right\rangle$. Let $\mathcal{M}=(M, g)$ be a pseudo-Riemannian manifold of signature $(p, q)$. Let $\mathcal{F}(M)$ be the bundle of frames $\vec{u}=\left(u_{1}, \ldots, u_{m}\right)$ for $T M$. Let $\mathcal{O}(\mathcal{M})$ and $\mathcal{H O}(\mathcal{M})$ be the sub-bundles:

$$
\begin{aligned}
& \mathcal{O}(\mathcal{M}):=\left\{\vec{u} \in \mathcal{F}(M): g\left(u_{i}, u_{j}\right)=\epsilon_{i j}\right\}, \\
& \mathcal{H O}(\mathcal{M}):=\left\{\vec{u} \in \mathcal{F}(M): g\left(u_{i}, u_{j}\right)=\lambda_{i j}^{\epsilon} \text { for some } 0<\lambda \in \mathbb{R}\right\} .
\end{aligned}
$$

Then $\mathcal{O}(M)$ is a principal $\mathcal{O}$ bundle while $\mathcal{H O}(M)$ is a principal $\mathcal{H O}$ bundle. We use the Levi-Civita connection, which is invariant under homothety transformations, to define a $\mathcal{H O}$ structure on $\mathcal{M}$ with a canonical connection.

Let $\mathfrak{h o}\left(T_{P} \mathcal{M}\right)$ be the Lie algebra of the group of homothety transformations of $T_{P} \mathcal{M}$. Let

$$
s_{0}:=\operatorname{dim}\left\{\mathfrak{h} \mathfrak{o}\left(T_{P} \mathcal{M}\right)\right\}=\frac{1}{2} m(m-1)+1 .
$$

For $0 \leq s \leq s_{0}$, we consider the subalgebras defined by:

$$
\begin{aligned}
& \mathfrak{h o}^{0}\left(T_{P} \mathcal{M}\right)=\left\{a \in \mathfrak{h o}\left(T_{P} \mathcal{M}\right) ; a \cdot R=0\right\}, \\
& \mathfrak{h o}^{s}\left(T_{P} \mathcal{M}\right)=\left\{a \in \mathfrak{h o}^{s-1}\left(T_{P} \mathcal{M}\right) ; a \cdot \nabla^{s} R=0\right\} .
\end{aligned}
$$

Clearly

$$
\mathfrak{h o}^{s}\left(T_{P} \mathcal{M}\right) \supset \mathfrak{h o}^{s+1}\left(T_{P} \mathcal{M}\right) \text { for all } s
$$

so we have a decreasing sequence of subalgebras of $\mathfrak{h o}\left(T_{P} \mathcal{M}\right)$. Let the Singer number $s(P)$ be the first integer stabilizing this sequence above, i.e.:

$$
\mathfrak{h o}^{s(P)+r}\left(T_{P} \mathcal{M}\right)=\mathfrak{h o}^{s(P)}\left(T_{P} \mathcal{M}\right) \text { for all } r \geq 1 \text {. }
$$

Now the assumption that $(\mathcal{M}, g)$ is homothety $k$-curvature homogeneous for some $k \geq \frac{1}{2} m(m-1)+1$ shows that the Singer number $s(P)$ is constant on $M$. The equivalences of Theorem 1.5 (2) now follow from the work of Podesta and Spiro [14]. (See also [13] for an extension to the affine setting.)

\section{The proof of Theorem 1.6}

Let $\mathcal{M}=(M, g)$ be a pseudo-Riemannian manifold which is homothety homogeneous with non-trivial homothety multiplicative character $\lambda$ and which is not VSI. Let $\mathcal{R}$ be a scalar Weyl invariant which does not vanish on $M$. Let $\mu_{\mathcal{R}}$ be as defined in Equation (1.b). We use Equation (1.a) to see that $\Phi^{*} \mu_{\mathcal{R}}=\lambda(\Phi) \mu_{\mathcal{R}}$ and thus $\Phi^{*} d \mu_{\mathcal{R}}=\lambda(\Phi) d \mu_{\mathcal{R}}$. Since $\mathcal{H}$ acts transitively on $M$, either $d \mu_{\mathcal{R}}$ never vanishes or $d \mu_{\mathcal{R}}$ vanishes identically; this latter possibility would imply $\mu_{\mathcal{R}}$ constant and hence $\lambda$ would be the trivial character which is false by assumption. Thus $d \mu_{\mathcal{R}}$ is never zero and the level sets $M_{c}^{\mathcal{R}}$ are smooth submanifolds of $M$ which have codimension 1. Furthermore,

$$
\Phi: M_{c}^{\mathcal{R}} \rightarrow M_{\lambda(\Phi) c}^{\mathcal{R}} \text { for any } \Phi \in \mathcal{H} .
$$

Since $\mathcal{H}$ acts transitively on $M, \mathcal{I}:=\operatorname{ker}(\lambda)$ acts transitively on $M_{c}^{\mathcal{R}}$. Consequently, $M_{1}^{\mathcal{R}}=\mathcal{I} \cdot P_{0}$ and $M_{c}^{\mathcal{R}}=\Phi \cdot \mathcal{I} \cdot P_{0}$ for any $\Phi \in \mathcal{H}$ with $\lambda(\Phi)=c$. This shows the level sets $M_{c}:=M_{c}^{\mathcal{R}}$ are in fact independent of the choice of $\mathcal{R}$ which establishes Assertion (1) of Theorem 1.6. 
We begin the proof of Theorem 1.6 (2) by establishing the following result:

Lemma 4.1. Let $\mathcal{M}=(M, g)$ be a Riemannian manifold which is homothety homogeneous with non-trivial homothety character $\lambda$ and which is not VSI.

(1) Let c sufficiently close to d. Let $P \in M_{c}$. There is a unique point $Q \in M_{d}$ which is the closest point to $P$ in $M_{d} ; d(P, Q)=d\left(M_{c}, M_{d}\right)$. If $\sigma$ is the shortest unit speed geodesic from $P$ to $Q$, then $\sigma$ is perpendicular to $M_{c}$ at $P$ and to $M_{d}$ at $Q$.

(2) Let $\sigma:[0, T] \rightarrow M$ be a unit speed geodesic which is perpendicular to $M_{\mu_{\mathcal{R}}(\sigma(0))}$ at $\sigma(0)$. Then $\sigma$ is perpendicular to $M_{\mu_{\mathcal{R}}(\sigma(t))}$ for any $t$ in the interval $[0, T]$. Furthermore, $\sigma\left[t_{0}, t_{1}\right]$ is a curve which minimizes the distance from $M_{\mu_{\mathcal{R}}\left(\sigma\left(t_{0}\right)\right)}$ to $M_{\mu_{\mathcal{R}}\left(\sigma\left(t_{1}\right)\right)}$ for any $0 \leq t_{0}<t_{1} \leq T$.

Proof. Choose $Q_{1} \in M_{d}$ to be a point on $M_{d}$ which is a closest point to $P$. There might, a-priori of course, be several such points. Let $\sigma_{1}$ be the unit speed geodesic from $P$ to $Q_{1}$ minimizing the distance so $\sigma_{1}(0)=P$ and $\sigma_{1}\left(t_{1}\right)=Q_{1}$. If $\dot{\sigma}\left(t_{1}\right)$ is not perpendicular to $T_{Q_{1}} M_{d}$, then we could "cut off the leg" to construct a point $Q_{2}$ of $M_{d}$ with $d\left(P, Q_{2}\right)<d\left(P, Q_{1}\right)$. Since this would contradict the choice of $Q_{1}$, we must have $\dot{\sigma}\left(t_{1}\right) \perp T_{Q_{1}} M_{d}$. Next suppose that $\dot{\sigma}(0)$ is not perpendicular to $T_{P} M_{c}$. Then we could "cut off the leg" to construct a point $P_{1} \in M_{c}$ so $d\left(P_{1}, Q_{1}\right)<d\left(P, Q_{1}\right)$. Note that $\mathcal{I}$ acts transitively on $M_{c}$ for any $c$. Choose $\phi \in \mathcal{I}$ so $\phi P_{1}=P$. Then:

$$
d\left(P, \phi Q_{1}\right)=d\left(\phi P_{1}, \phi Q_{1}\right)=d\left(P_{1}, Q_{1}\right)<d\left(P, Q_{1}\right)
$$

which again contradicts the choice of $Q_{1}$. This shows that the closest point is unique. Given any other point $P_{2} \in M_{c}$, we construct $Q_{2}$ similarly. Choose an isometry $\phi \in \mathcal{I}$ with $\phi P_{2}=P$. Then we must have that $\phi Q_{2}=Q$ and thus $d(P, Q)=d\left(P_{2}, Q_{2}\right)$. This proves Assertion (1); Assertion (2) is an immediate consequence of Assertion (1).

We now prove Theorem 1.6 (2). Suppose $1<s_{1}<s_{2}$. Choose homotheties $\Phi_{i}$ so $\lambda\left(\Phi_{i}\right)=s_{i}$. We then have

$$
\Phi_{1} M_{1}=M_{s_{1}}, \quad \Phi_{2} M_{1}=M_{s_{2}}, \quad\left(\Phi_{1} \Phi_{2}\right) M_{1}=\Phi_{1} M_{s_{2}}=\Phi_{2} M_{s_{1}}=M_{s_{1} s_{2}} .
$$

Therefore,

$$
\begin{aligned}
& d\left(M_{1}, M_{s_{1} s_{2}}\right)=d\left(M_{1}, M_{s_{1}}\right)+d\left(M_{s_{1}}, M_{s_{1} s_{2}}\right) \\
= & d\left(M_{1}, M_{s_{1}}\right)+d\left(\Phi_{1} M_{1}, \Phi_{1} M_{s_{2}}\right)=d\left(M_{1}, M_{s_{1}}\right)+\lambda\left(\Phi_{1}\right) d\left(M_{1}, M_{s_{2}}\right) \\
= & d\left(M_{1}, M_{s_{1}}\right)+s_{1} d\left(M_{1}, M_{s_{2}}\right) .
\end{aligned}
$$

Similarly, we have that $d\left(M_{1}, M_{s_{1} s_{2}}\right)=d\left(M_{1}, M_{s_{2}}\right)+s_{2} d\left(M_{1}, M_{s_{1}}\right)$. Thus

$$
d\left(M_{1}, M_{s_{1}}\right)+s_{1} d\left(M_{1}, M_{s_{2}}\right)=d\left(M_{1}, M_{s_{2}}\right)+s_{2} d\left(M_{1}, M_{s_{1}}\right) .
$$

Consequently $d\left(M_{1}, M_{s_{1}}\right)\left(s_{2}-1\right)=d\left(M_{1}, M_{s_{2}}\right)\left(s_{1}-1\right)$ so

$$
\kappa:=\frac{d\left(M_{1}, M_{s_{1}}\right)}{s_{1}-1}=\frac{d\left(M_{1}, M_{s_{2}}\right)}{s_{2}-1}
$$

is independent of the choice of $s_{1}$ and $s_{2}$ for $1<s_{1}<s_{2}$. Let $s<t$. Choose a homothety $\Phi$ so $\lambda(\Phi)=s$. Then $\Phi\left(M_{\frac{t}{s}}\right)=M_{t}$. Since $1<\frac{t}{s}$,

$$
d\left(M_{s}, M_{t}\right)=d\left(\Phi M_{1}, \Phi M_{\frac{t}{s}}\right)=s d\left(M_{1}, M_{\frac{t}{s}}\right)=s \kappa\left(\frac{t}{s}-1\right)=\kappa(t-s) .
$$

Let $0<c<1$. Then $d\left(M_{c}, M_{1}\right)=\kappa(1-c) \leq \kappa$. Let $\sigma$ be a unit speed geodesic with $\sigma(0) \in M_{1}$, with $\dot{\sigma}(0) \perp M_{1}$, and with $g\left(\dot{\sigma}(0), \operatorname{grad} \mu_{\mathcal{R}}\right)=-1$. By Lemma 4.1 $d\left(\sigma(t), M_{1}\right)=t$. Consequently $t<\kappa$ and the geodesic $\sigma$ does not extend for infinite time. This shows $\mathcal{M}$ is incomplete which completes the proof of Theorem 1.6 . 


\section{The Proof of Theorem 1.8}

We adopt the notation of Definition 1.7 and let $\mathcal{P}=\left(M, g_{0}, \mathcal{H}, \lambda\right)$ be an $\mathfrak{P}$ structure. Let $P_{0}$ be the base point of $M$ and let $\mathcal{H}_{0}:=\left\{\Phi \in \mathcal{H}: \Phi P_{0}=P_{0}\right\}$ be the isotropy group. By assumption $\mathcal{H}_{0} \subset \operatorname{ker}(\lambda)$ so $M_{1}:=\operatorname{ker}(\lambda) / \mathcal{H}_{0}=\lambda^{-1}(\{1\}) / \mathcal{H}_{0}$ is a smooth submanifold of $M=\mathcal{H} / \mathcal{H}_{0}$ of codimension 1 . Choose a smooth 1 parameter subgroup $\Phi_{t}$ of $\mathcal{H}$ (written multiplicatively) so that $\lambda\left(\Phi_{t}\right)=t$ for $t \in \mathbb{R}^{+}$. This permits us to decompose

$$
\mathcal{H}=\mathbb{R}^{+} \times \operatorname{ker}(\lambda)
$$

as a differentiable manifold; the multiplicative character $\lambda$ is then projection on the first factor. The decomposition of Equation (5.a) will not, of course, in general preserve the group structure; we shall return to this point in Section 6. Equation (5.a) induces a corresponding decomposition

$$
M=\mathcal{H} / \mathcal{H}_{0}=\mathbb{R}^{+} \times \operatorname{ker}(\lambda) / \mathcal{H}_{0}=\mathbb{R}^{+} \times M_{1} .
$$

The slice $M_{t}:=\Phi_{t} M_{1}$ corresponds to $\{t\} \times M_{1}$. We have $g_{\lambda}(t, y)=t^{2} g(t, y)$. We compute that

$$
\begin{aligned}
& \left\{\Phi_{s}^{*}\left(g_{\lambda}\right)(t, y)\right\}\left(\xi_{1}, \xi_{2}\right)=\left\{g_{\lambda}(s t, y)\right\}\left(\left(\Phi_{s}\right)_{*} \xi,\left(\Phi_{s}\right)_{*} \xi_{2}\right) \\
= & s^{2} t^{2}\left\{g_{0}(s t, y)\right\}\left(\left(\Phi_{s}\right)_{*} \xi,\left(\Phi_{s}\right)_{*} \xi_{2}\right)=s^{2} t^{2} g_{0}(t, y)\left(\xi_{1}, \xi_{2}\right)=s^{2} g_{\lambda}(t, y)\left(\xi_{1}, \xi_{2}\right)
\end{aligned}
$$

and thus $\Phi_{s}^{*}\left(g_{\lambda}\right)=s^{2} g_{\lambda}$. On the other hand, if $\Phi \in \operatorname{ker}(\lambda)$, then

$$
\begin{aligned}
& \left\{\Phi^{*}\left(g_{\lambda}\right)(t, y)\right\}\left(\xi_{1}, \xi_{2}\right)=\left\{g_{\lambda}(t, \Phi y)\right\}\left(\Phi_{*} \xi_{1}, \Phi_{*} \xi_{2}\right) \\
= & t^{2} g_{0}(t, \Phi y)\left(\Phi_{*} \xi_{1}, \Phi_{*} \xi_{2}\right)=t^{2} g_{0}(t, y)\left(\xi_{1}, \xi_{2}\right)=g_{\lambda}(t, y)\left(\xi_{1}, \xi_{2}\right)
\end{aligned}
$$

and thus $\Phi^{*} g_{\lambda}=g_{\lambda}$. This implies that $\Phi$ acts by isometries on $\left(M, g_{\lambda}\right)$. Since we can decompose any $\Phi \in \mathcal{H}$ in the form $\Phi=\Phi_{t} \Psi$ for $\Psi \in \operatorname{ker}(\lambda)$, we conclude $\Phi^{*} g_{\lambda}=\lambda(\Phi)^{2} g_{\lambda}$ for any $\Phi \in \mathcal{H}$. Since $\mathcal{H}$ acts transitively on $M$, this implies $\left(M, g_{\lambda}\right)$ is homothety homogeneous with homothety character $\lambda$ and $\Phi: M_{t} \rightarrow M_{\lambda(\Phi) t}$.

Conversly, if $(M, g)$ is homothety homogeneous with non-trivial homothety character $\lambda$ and if $(M, g)$ is not a VSI manifold, then we can use Theorem [1.6] to find the level sets $M_{c}$. We may define $g_{0}(P):=\mu_{\mathcal{R}}(P)^{-2} g(P)$ to define a conformally equivalent manifold on which $\mathcal{H}$ acts by isometries. The corresponding $\mathfrak{P}$ structure is then given by $\left(M, g_{0}, \mathcal{H}, \lambda\right)$. The fact that the isotropy subgroup $H_{0} \subset \operatorname{ker}(\lambda)$ then follows; the fact that $\mathcal{M}$ is not VSI plays a crucial role in defining the level sets.

\section{The proof of Theorem 1.11}

Let $\mathcal{Q}=\left(N, g_{N}, \mathcal{I}, \theta\right)$ be a $\mathfrak{Q}$ structure. Fix a point $P \in N$; which point is chosen is irrelevant since $\left(N, g_{N}\right)$ is a homogeneous space. If $\theta=0$, then $g_{t, \mathcal{Q}}$ is non-singular so we suppose $\theta \neq 0$. First suppose that $\theta$ is not a null covector. Choose an orthonormal basis $\left\{\eta_{2}, \ldots, \eta_{m}\right\}$ for $T_{P} N$ so that $\theta\left(\eta_{i}\right)=0$ for $i \geq 3$, so that $\theta\left(\eta_{2}\right)=c$, and so that $g\left(\eta_{i}, \eta_{j}\right)=\epsilon_{i} \delta_{i j}$ where $\epsilon_{i}= \pm 1$ for $2 \leq i, j \leq m$. Let $\eta_{1}=\partial_{x}$. We then have:

$$
\operatorname{det}\left(g_{i j}\right)=e^{m t x} \operatorname{det}\left(\begin{array}{ll}
1 & c \\
c & \epsilon_{2}
\end{array}\right) \cdot \prod_{i \geq 3} \epsilon_{i} .
$$

If $\epsilon_{2}=-1$, then $\operatorname{det}\left(g_{i j}\right) \neq 0$. In this setting $\theta$ is timelike so $g_{N}(\theta, \theta)<0$. If $\epsilon_{2}=+1$, then $\theta$ is spacelike and the metric is non-degenerate if and only if $c^{2} \neq 1$ or equivalently $g_{N}(\theta, \theta) \neq 1$. Suppose $0 \neq \theta$ is a null covector. We can choose 
an orthonormal basis $\left\{\eta_{2}, \ldots, \eta_{m}\right\}$ for $T_{P} N$ so $\eta_{2}$ is spacelike, so $\eta_{3}$ is timelike, so $\theta\left(\eta_{2}\right)=\theta\left(\eta_{3}\right)=c$, and so $\theta\left(\eta_{i}\right)=0$ for $i \geq 4$. We then have

$$
\operatorname{det}\left(g_{i j}\right)=e^{m t x} \operatorname{det}\left(\begin{array}{rrr}
1 & c & c \\
c & 1 & 0 \\
c & 0 & -1
\end{array}\right) \cdot \prod_{i \geq 4} \epsilon_{i}=e^{m t x}(-1-c(-c)+c(-c)) \neq 0 .
$$

The map $x \rightarrow e^{x}$ provides a group isomorphism between $(\mathbb{R},+)$ and $\left(\mathbb{R}^{+}, \cdot\right)$ which we use to write the character additively and express $\mathcal{H}=\mathbb{R} \times \operatorname{ker}(\lambda)$. If $\Phi=(a, \phi) \in \mathcal{H}$, let $\Phi:(x, y) \rightarrow(x+a, \phi y)$ define a transitive action of $\mathcal{H}$ on $M=\mathbb{R} \times N$. Since $\theta$ is an invariant 1-form, we have $\Phi^{*} g_{t, \mathcal{Q}}=e^{t a} g_{t, \mathcal{Q}}$. Assertion (1) now follows.

Conversly, suppose that $\mathcal{M}$ is a homothety homogeneous manifold with nontrivial homothety character which is not VSI. Suppose the homothety character $\lambda$ splits. We normalize the homothety vector field $\mathfrak{h}$ (which is assumed to be spacelike) so that $g_{M}(\mathfrak{h}, \mathfrak{h})=1$ on $M_{1}$. We now write the flow additively to construct a diffeomorphism of $M$ with $\mathbb{R} \times M_{1}$. Let $\theta(\eta):=g_{M}(\mathfrak{h}, \eta)$ for $\eta \in T M_{1}$. Since $\mathfrak{h}$ is invariant under the action of $\mathcal{I}=\operatorname{ker}(\lambda), \theta$ is an invariant 1-form on $M_{1}$ and the metric for any point of $M_{1}$ takes the form $g_{M}=d x^{2}+d x \circ \theta+g_{M_{1}}$. Using $\Phi_{x}$ to push the metric from $M_{1}$ to $M_{e^{x} 1}$ then introduces the desired conformal factor for some $t$.

\section{The Proof of TheOrem 1.12}

Suppose that $\mathcal{M}=\mathcal{M}_{t, \mathfrak{Q}}$ is isomorphic to $\mathcal{M}_{\tilde{t}, \tilde{\mathcal{Q}}}$ for some $\tilde{\mathcal{Q}}$ structure with $\tilde{\theta}=0$. Then there exists a homothety vector field $\tilde{\mathfrak{h}}$ on $\mathcal{M}$ which is central and which is perpendicular to the slices. Thus we can write $\mathfrak{h}=\tilde{\mathfrak{h}}+\xi$ where $\xi$ is a killing vector field. We then have $\theta(\eta)=g_{M}(\tilde{\mathfrak{h}}, \eta)+g_{M}(\xi, \eta)=g_{M}(\xi, \eta)$ and thus $\theta$ is dual to $\xi$ with respect to the metric $g_{N}$ on the slice $\{0\} \times N$. Consequently $\theta_{a ; b}+\theta_{b ; a}=0$.

Next suppose that $\theta_{a ; b}+\theta_{b ; a}=0$. The associated dual vector field $\xi$ is then a Killing vector field which is invariant under the action of $\mathcal{I}$. Since $\mathcal{I}$ acts transitively on $N$, we can integrate $\xi$ to find a smooth 1-parameter flow $\left\{\phi_{\epsilon}\right\}_{\epsilon \in \mathbb{R}}$ of isometries which commutes with $\mathcal{I}$. Let $\tilde{\mathcal{I}}$ be the (possibly larger) group of isometries generated by $\mathcal{I}$ and $\left\{\phi_{\epsilon}\right\}_{\epsilon \in \mathbb{R}}$. Let $\tilde{\mathcal{Q}}:=\left(N, g_{N}, \tilde{\mathcal{I}}, 0\right)$ be the extended $\mathfrak{Q}$ structure. Let

$$
\varrho:=\left(1-\|\xi\|^{2}\right)^{-1 / 2} \text { and } s:=\varrho t .
$$

Set $\tilde{\mathcal{M}}_{s}=\mathcal{M}_{s, \tilde{\mathfrak{Q}}}$. Define a diffeomorphism $\Psi$ of $M=\mathbb{R} \times N$ by setting

$$
\Psi_{\varrho}(x, y):=\left(x, \phi_{\varrho x} y\right) .
$$

We will show that $\Psi_{\varrho}$ is an isometry from $\mathcal{M}$ to $\tilde{\mathcal{M}}_{s}$. Fix $(x, y) \in M$. Let $\eta \in T_{y} N$. We compute:

$$
\Psi_{*} \partial_{x}=\partial_{x}+\varrho \phi_{\varrho x, *} \xi \text { and } \Psi_{*} \eta=\phi_{\varrho x, *} \eta
$$

Since $\phi_{\varrho x, *}$ is an isometry, we have:

$$
\begin{aligned}
& g_{\tilde{M}_{s}}\left(\Psi_{*} \partial_{x}, \Psi_{*} \partial_{x}\right)=e^{s x}\left\{1+\varrho^{2}\|\xi\|^{2}\right\}, \\
& g_{\tilde{M}_{s}}\left(\Psi_{*} \partial_{x}, \Psi_{*} \eta\right)=e^{s x} \varrho \theta(\eta), \\
& g_{\tilde{M}_{s}}\left(\left(\Psi_{*} \eta_{1}, \Psi_{*} \eta_{2}\right)\right)=e^{s x} g_{N}\left(\eta_{1}, \eta_{2}\right), \\
& \Psi^{*} g_{s, \tilde{\mathcal{Q}}}=e^{s x}\left(\left(1+\varrho^{2}\|\xi\|^{2}\right) d x^{2}+\varrho d x \circ \theta+g_{N}\right) .
\end{aligned}
$$

We use a diffeomorphism $\Upsilon$ to change variables setting $\tilde{x}=\left(1+\rho^{2}\|\xi\|^{2}\right)^{1 / 2} x$ and $\tilde{t}=\left(1+\rho^{2}\|\xi\|^{2}\right)^{-1 / 2} s$. Then:

$$
\Upsilon^{*} g_{\tilde{M}_{s}}=e^{\tilde{t} \tilde{x}}\left(d \tilde{x}^{2}+\varrho\left(1+\rho^{2}\|\xi\|^{2}\right)^{-1 / 2} d \tilde{x} \circ \theta+g_{N}\right) .
$$


We use the defining relation for $\varrho$ to see

$$
\begin{aligned}
& \varrho\left(1+\varrho^{2}\|\xi\|^{2}\right)^{-1 / 2}=\left\{\frac{1}{1-\|\xi\|^{2}}\right\}^{1 / 2}\left\{1+\frac{\|\xi\|^{2}}{1-\|\xi\|^{2}}\right\}^{-1 / 2}=1, \\
& \tilde{t}=\left(1+\rho^{2}\|\xi\|^{2}\right)^{-1 / 2} s=\left(1+\frac{\|\xi\|^{2}}{1-\|\xi\|^{2}}\right)^{-1 / 2} s=\left(1-\|\xi\|^{2}\right)^{1 / 2} s=t .
\end{aligned}
$$

We use the variable $\tilde{x}$ instead of $x$ to see that $\Psi$ provides an isometry between $\mathcal{M}$ and $\mathcal{M}_{s, \tilde{\mathcal{Q}}}$.

\section{The PROOF OF THEOREM 1.13}

Let $\mathcal{M}=\mathcal{M}_{t, \mathcal{Q}}$ where $\mathcal{Q}=\left(N, g_{N}, \mathcal{I}, \theta\right)$ is a $\mathfrak{Q}$ structure with $\theta=0$. We examine the curvature tensor. Fix $t$ and fix a point $P \in N$. Let $g=g_{N}$ and let $\tilde{g}=g_{t, \mathcal{Q}}$. Choose local coordinates $y=\left(y^{1}, \ldots, y^{m-1}\right)$ centered at $P$. Let indices $u, v, w$ range from 0 to $m-1$ and index the coordinate frame $\left(\partial_{x}, \partial_{y_{1}}, \ldots, \partial_{y_{m-1}}\right)$; indices $i, j, k$ range from 1 to $m-1$ and index the coordinate frame $\left(\partial_{y_{1}}, \ldots, \partial_{y_{m-1}}\right)$. Let $\Gamma$ be the Christoffel symbols of $g$ and $\tilde{\Gamma}$ be the Christoffel symbols of $\tilde{g}$. Let $\delta_{i}^{j}$ be the Kronecker index. We compute:

$$
\begin{array}{lll}
\tilde{g}_{00}=e^{t x}, & \tilde{g}_{0 i}=0, \quad \tilde{g}_{i j}=e^{t x} g_{i j}, \\
\tilde{\Gamma}_{00}{ }^{0}=\frac{1}{2} t & \tilde{\Gamma}_{00}{ }^{i}=0, & \\
\tilde{\Gamma}_{0 i}{ }^{0}=0, & \tilde{\Gamma}_{0 i}{ }^{k}=\frac{1}{2} t \delta_{i}^{k} & \\
\tilde{\Gamma}_{i j}{ }^{0}=-\frac{1}{2} t g_{i j}, & \tilde{\Gamma}_{i j}{ }^{k}=\Gamma_{i j}{ }^{k} .
\end{array}
$$

Thus the covariant derivatives are given by

$$
\begin{array}{ll}
\tilde{\nabla}_{\partial_{x}} \partial_{x}=\frac{1}{2} t \partial_{x}, & \tilde{\nabla}_{\partial_{x}} \partial_{y_{i}}=\frac{1}{2} t \partial_{y_{i}}, \\
\tilde{\nabla}_{\partial_{y_{i}}} \partial_{x}=\frac{1}{2} t \partial_{y_{i}}, & \tilde{\nabla}_{\partial_{y_{i}}} \partial_{y_{j}}=\Gamma_{i j}{ }^{k} \partial_{y_{k}}-\frac{1}{2} t g_{i j} \partial_{x} .
\end{array}
$$

We choose the coordinate system so the first derivatives of $g_{i j}$ vanish at $P$ and hence $\Gamma(P)=0$. Consequently the curvature operator at $P$ is given by:

$$
\begin{aligned}
\tilde{\mathcal{R}}\left(\partial_{x}, \partial_{y_{i}}\right) \partial_{x} & =\left\{\tilde{\nabla}_{\partial_{x}} \tilde{\nabla}_{\partial_{y_{i}}}-\tilde{\nabla}_{\partial_{y_{i}}} \tilde{\nabla}_{\partial_{x}}\right\} \partial_{x}=\frac{1}{2} t \tilde{\nabla}_{\partial_{x}} \partial_{y_{i}}-\frac{1}{2} t \tilde{\nabla}_{\partial_{y_{i}}} \partial_{x}=0, \\
\tilde{\mathcal{R}}\left(\partial_{x}, \partial_{y_{i}}\right) \partial_{y_{j}} & =\left\{\tilde{\nabla}_{\partial_{x}} \tilde{\nabla}_{\partial_{y_{i}}}-\tilde{\nabla}_{\partial_{y_{i}}} \tilde{\nabla}_{\partial_{x}}\right\} \partial_{y_{j}} \\
& =\tilde{\nabla}_{\partial_{x}}\left\{\Gamma_{i j}{ }^{k} \partial_{y_{k}}-\frac{1}{2} t g_{i j} \partial_{x}\right\}-\frac{1}{2} t \tilde{\nabla}_{\partial_{y_{i}}} \partial_{y_{j}} \\
& =\frac{1}{2} t\left\{\Gamma_{i j}^{k} \partial_{y_{k}}-\frac{1}{2} t g_{i j} \partial_{x}\right\}-\frac{1}{2} t\left\{\Gamma_{i j}{ }^{k} \partial_{y_{k}}-\frac{1}{2} t g_{i j} \partial_{x}\right\}=0, \\
\tilde{\mathcal{R}}\left(\partial_{y_{i}}, \partial_{y_{j}}\right) \partial_{x} & =\left\{\tilde{\nabla}_{\partial_{y_{i}}} \tilde{\nabla}_{\partial_{y_{j}}}-\tilde{\nabla}_{\partial_{y_{j}}} \tilde{\nabla}_{\partial_{y_{i}}}\right\} \partial_{x}=\frac{1}{2} t \tilde{\nabla}_{\partial_{y_{i}}} \partial_{y_{j}}-\frac{1}{2} t \tilde{\nabla}_{\partial_{y_{j}}} \partial_{y_{i}}=0, \\
\tilde{\mathcal{R}}\left(\partial_{y_{i}}, \partial_{y_{j}}\right) \partial_{y_{k}} & =\left\{\tilde{\nabla}_{\partial_{y_{i}}} \tilde{\nabla}_{\partial_{y_{j}}}-\tilde{\nabla}_{\partial_{y_{j}}} \tilde{\nabla}_{\partial_{y_{i}}}\right\} \partial_{y_{k}} \\
& =\tilde{\nabla}_{\partial_{y_{i}}}\left(\Gamma_{j k}^{\ell} \partial_{y_{\ell}}-\frac{1}{2} t g_{j k} \partial_{x}\right)-\tilde{\nabla}_{\partial_{y_{j}}}\left(\Gamma_{i k}^{\ell} \partial_{y_{\ell}}-\frac{1}{2} t g_{i k} \partial_{x}\right) \\
& =R_{i j k} \partial_{y_{\ell}}-\frac{1}{4} t^{2} g_{j k} \partial_{y_{i}}+\frac{1}{4} t^{2} g_{i k} \partial_{y_{j}} .
\end{aligned}
$$

We can now express the scalar curvature and Ricci tensor $\{\tilde{\rho}, \tilde{\tau}\}$ for $\tilde{g}$ in terms of the scalar curvature and Ricci tensor $\{\rho, \tau\}$ for $g$ and complete the proof of Theorem 1.13 (4) by computing:

$$
\tilde{\rho}=\rho-\frac{m-2}{4} t^{2} g \quad \text { and } \quad \tilde{\tau}=e^{-t x}\left\{\tau-\frac{(m-1)(m-2)}{4} t^{2}\right\}
$$




\section{The Proof of Theorem 1.16}

Let $\mathcal{O}$ be a connected open subset of $\mathbb{R}^{3}$, let $f=f(x, y) \in C^{\infty}(\mathcal{O})$, and let $\mathcal{M}_{f}=\left(\mathcal{O}, g_{f}\right)$ where

$$
g_{f}\left(\partial_{x}, \partial_{x}\right)=-2 f(x, y), \quad g_{f}\left(\partial_{x}, \partial_{\tilde{x}}\right)=g_{f}\left(\partial_{y}, \partial_{y}\right)=1 .
$$

We suppress the subscript " $f$ " when no confusion is likely to ensure. We follow the discussion in [8]. The (possibly) non-zero covariant derivatives are given by:

$$
\nabla_{\partial_{x}} \partial_{x}=-f_{x} \partial_{\tilde{x}}+f_{y} \partial_{y} \text { and } \nabla_{\partial_{x}} \partial_{y}=\nabla_{\partial_{y}} \partial_{x}=-f_{y} \partial_{\tilde{x}} \text {. }
$$

This shows that $\operatorname{Range}(\mathcal{R}) \subset \operatorname{Span}\left\{\partial_{y}, \partial_{\tilde{x}}\right\}$. Covariantly differentiating this relationship implies similarly Range $\left(\nabla^{k} \mathcal{R}\right) \subset \operatorname{Span}\left\{\partial_{y}, \partial_{\tilde{x}}\right\}$. for any $k$. Furthermore, $\nabla^{k} \mathcal{R}(\cdot)$ vanishes if any entry is $\partial_{\tilde{x}}$ since $\nabla_{\tilde{x}}=0$ and since the metric is independent of $\tilde{x}$. Lowering indices then shows that $\nabla^{k} R(\cdot)=0$ if any index is $\partial_{\tilde{x}}$. Thus the only non-zero entries take the form $\nabla^{k} R\left(\partial_{x}, \partial_{y}, \partial_{y}, \partial_{x} ; \ldots\right)$. Relative to the basis $\left\{\partial_{x}, \partial_{\tilde{x}}, \partial_{y}\right\}$, the metric tensor $g_{i j}$ and the inverse metric tensor $g^{i j}$ are given by:

$$
\left(g_{i j}\right)=\left(\begin{array}{rrr}
-2 f & 1 & 0 \\
1 & 0 & 0 \\
0 & 0 & 1
\end{array}\right) \text { and }\left(g^{i j}\right)=\left(\begin{array}{rrr}
0 & 1 & 0 \\
1 & 2 f & 0 \\
0 & 0 & 1
\end{array}\right) .
$$

Thus any Weyl contraction must involve a $\partial_{\tilde{x}}$ variable; the curvature tensor vanishes on such variables and thus these manifolds are VSI. This establishes Theorem 1.14,

We now establish Theorem 1.16. The (possibly) non-zero curvatures and covariant derivatives to order 2 are:

$$
\begin{aligned}
& R\left(\partial_{x}, \partial_{y}, \partial_{y}, \partial_{x}\right)=f_{y y} \\
& \nabla R\left(\partial_{x}, \partial_{y}, \partial_{y}, \partial_{x} ; \partial_{x}\right)=f_{x y y} \\
& \nabla R\left(\partial_{x}, \partial_{y}, \partial_{y}, \partial_{x} ; \partial_{y}\right)=f_{y y y} \\
& \nabla^{2} R\left(\partial_{x}, \partial_{y}, \partial_{y}, \partial_{x} ; \partial_{x}, \partial_{x}\right)=f_{x x y y}-f_{y} f_{y y y} \\
& \nabla^{2} R\left(\partial_{x}, \partial_{y}, \partial_{y}, \partial_{x} ; \partial_{x}, \partial_{y}\right)=\nabla^{2} R\left(\partial_{x}, \partial_{y}, \partial_{y}, \partial_{x} ; \partial_{y}, \partial_{x}\right)=f_{x y y y} \\
& \nabla^{2} R\left(\partial_{x}, \partial_{y}, \partial_{y}, \partial_{x} ; \partial_{y}, \partial_{y}\right)=f_{y y y y}
\end{aligned}
$$

If $f_{y y}$ vanishes identically, then $\mathcal{M}$ is flat. The vanishing of $f_{y y}$ is an invariant of the homothety 0 -model. Since we are interested in homothety curvature homogeneity, we shall assume $f_{y y}$ never vanishes; since $M$ is connected, either $f_{y y}$ is always positive or $f_{y y}$ is always negative. We shall usually assume $f_{y y}>0$ as the other case is handled similarly. The simultaneous vanishing of $f_{y y y}$ and of $f_{x y y}$ is an invariant of the homothety 1 -model. The case $f_{y y}=a$ for $0 \neq a \in \mathbb{R}$ gives rise to a symmetric space. We shall therefore assume $f_{y y}$ non-constant. This gives rise to two cases $f_{y y y}$ never zero and $f_{y y y}$ vanishing identically but $f_{x y y}$ never zero.

Definition 9.1. Let $\mathfrak{M}_{1, c_{1}, c_{2}}$ be the 1-curvature model whose (possibly) non-zero components are defined by $\varepsilon_{13}=\varepsilon_{22}=1, c_{1221}=1, c_{12211}=c_{1}$, and $c_{12212}=c_{2}$ :

$$
\begin{array}{lll}
\left\langle\xi_{1}, \xi_{3}\right\rangle=1, & \left\langle\xi_{2}, \xi_{2}\right\rangle=1, & R\left(\xi_{1}, \xi_{2}, \xi_{2}, \xi_{1}\right)=1, \\
\nabla R\left(\xi_{1}, \xi_{2}, \xi_{2}, \xi_{1} ; \xi_{1}\right)=c_{1}, & \nabla R\left(\xi_{1}, \xi_{2}, \xi_{2}, \xi_{1} ; \xi_{2}\right)=c_{2} .
\end{array}
$$

9.1. Proof of Theorem $\mathbf{1 . 1 6}(\mathbf{1 , 2})$. We suppose $f_{y y}>0$. The distributions

$$
\operatorname{ker}(\Re)=\operatorname{Span}\left\{\partial_{\tilde{x}}\right\} \text { and } \operatorname{Range}(\mathfrak{R})=\operatorname{Span}\left\{\partial_{y}, \partial_{\tilde{x}}\right\}
$$

are invariantly defined. To preserve these distributions, we set:

$$
\xi_{1}=a_{11}\left(\partial_{x}+f \partial_{\tilde{x}}+a_{12} \partial_{y}+a_{13} \partial_{\tilde{x}}\right), \quad \xi_{2}=\partial_{y}+a_{23} \partial_{\tilde{x}}, \quad \xi_{3}=a_{33} \partial_{\tilde{x}},
$$

for some functions $a_{i j}$ on $\mathcal{O}$. To ensure that the inner products are normalized properly, we impose the relations:

$$
a_{12}^{2}+2 a_{13}=0, \quad a_{12}+a_{23}=0, \quad a_{11} a_{33}=1 .
$$


This determines $a_{13}, a_{23}$, and $a_{33}$; these parameters play no further role and $\left\{\lambda, a_{11}, a_{12}\right\}$ remain as free parameters where $\lambda$ is the homothety rescaling factor. We suppose $f_{y y y} \neq 0$. Set:

$$
\lambda:=f_{y y y} f_{y y}^{-1}, \quad a_{12}:=-f_{x y y} f_{y y y}^{-1}, \quad a_{11}^{2}:=f_{y y}^{-1} \lambda^{2} .
$$

We then have

$$
\begin{aligned}
& R\left(\xi_{1}, \xi_{2}, \xi_{2}, \xi_{1}\right)=a_{11}^{2} f_{y y}=\lambda^{2}, \\
& \nabla R\left(\xi_{1}, \xi_{2}, \xi_{2}, \xi_{1} ; \xi_{1}\right)=a_{11}^{3}\left\{f_{x y y}+a_{12} f_{y y y}\right\}=0, \\
& \nabla R\left(\xi_{1}, \xi_{2}, \xi_{2}, \xi_{1} ; \xi_{2}\right)=a_{11}^{2} f_{y y y}=\lambda^{2} f_{y y}^{-1} f_{y y y}=\lambda^{3} .
\end{aligned}
$$

All the parameters of the theory have been determined (modulo a possible sign ambiguity in $a_{11}$ ) and any homothety 1 -model for $\mathcal{M}_{f}$ is isomorphic to $\mathfrak{M}_{1,0,1}$ in this special case. This proves Theorem 1.16 (1).

Suppose $f_{y y}>0, f_{y y y}=0$, and $f_{x y y}$ never vanishes. Set $f_{y y}=\alpha(x)$. The parameter $a_{12}$ plays no role. To ensure that $\mathcal{M}_{f}$ is homothety 1-curvature homogeneous, we impose the following relations where $\left\{a_{11}, \lambda\right\}$ are unknown functions to be determined and where $\left\{c_{0}, c_{1}\right\}$ are unknown constants:

$$
\begin{aligned}
& R\left(\xi_{1}, \xi_{2}, \xi_{2}, \xi_{1}\right)=a_{11}^{2}(x) \alpha(x)=\lambda^{2}(x) c_{0}, \\
& R\left(\xi_{1}, \xi_{2}, \xi_{2}, \xi_{1} ; \xi_{1}\right)=a_{11}^{3}(x) \alpha_{x}(x)=\lambda^{3}(x) c_{1}, \\
& R\left(\xi_{1}, \xi_{2}, \xi_{2}, \xi_{1} ; \xi_{2}\right)=0 .
\end{aligned}
$$

Consequently, $a_{11}^{6}(x) \alpha^{3}(x)=\lambda^{6}(x) c_{0}^{3}$ and $a_{11}^{6}(x) \alpha_{x}^{2}(x)=\lambda^{6}(x) c_{1}^{2}$. This shows that $\alpha^{3}(x)=c_{3} \alpha_{x}^{2}(x)$ for some constant $c_{3}$. We solve this ordinary differential equation to see that

$$
\alpha(x)=a\left(x-x_{0}\right)^{-2} \text { for } 0 \neq a \in \mathbb{R} \text { and } x_{0} \in \mathbb{R} .
$$

This has the form given in Theorem $1.15(2 \mathrm{~b})$ and defines a locally homogeneous example. Theorem 1.16 (2) now follows.

9.2. The proof of Theorem $\mathbf{1 . 1 6}$ (3). We assume that $f_{y y}$ and $f_{y y y}$ never vanish as this case is the only possible source of new examples not covered by Theorem 1.15] We shall suppose $f_{y y}>0$; the case $f_{y y}<0$ is handled similarly. As any two homothety 1-curvature models for $\mathcal{M}_{f}$ are isomorphic, we can adopt the normalizations of Equation (9.a), (9.b), and (9.c). We have:

$$
\begin{aligned}
& R\left(\xi_{1}, \xi_{2}, \xi_{2}, \xi_{1}\right)=a_{11}^{2} f_{y y}=\lambda^{2}, \\
& \nabla R\left(\xi_{1}, \xi_{2}, \xi_{2}, \xi_{1} ; \xi_{2}\right)=a_{11}^{2} f_{y y y}=\lambda^{3}, \\
& \nabla^{2} R\left(\xi_{1}, \xi_{2}, \xi_{2}, \xi_{1} ; \xi_{2}, \xi_{2}\right)=a_{11}^{2}(x) f_{y y y y}=\lambda^{4} c_{122122}, \\
& \frac{f_{y y} \cdot f_{y y y y}}{f_{y y y} \cdot f_{y y y}}=\frac{\lambda^{2} a_{11}^{-2} \cdot \lambda^{4} c_{11} a_{11}^{-2}}{\lambda^{6} a_{11}^{-4}}=c_{122122} .
\end{aligned}
$$

Thus $c_{122122}$ is an invariant of the theory; this will imply the 3 families of the theory fall into different local isometry types. The ordinary differential equation $\frac{\alpha \alpha^{\prime \prime}}{\alpha^{\prime} \alpha^{\prime}}=c_{122122}$ has the solutions $\alpha>0$ (see, for example, Lemma 1.5.5 of [9]) of the form:

$$
\alpha(t)=\left\{\begin{array}{ll}
e^{a(t+b)} & \text { if } c_{122122}=1 \\
a(t+b)^{c} & \text { if } c_{122122} \neq 1
\end{array}\right\} \text { for } a \neq 0 \text { and } c \neq 0
$$

Thus

$$
f_{y y}=\left\{\begin{array}{ll}
e^{\alpha(x)(y+\beta(x))} & \text { if } c_{122122}=1 \text { for } \alpha(x) \neq 0 \\
\alpha(x)(y+\beta(x))^{c} & \text { if } c_{122122} \neq 1 \text { for } \alpha(x) \neq 0 \text { and } c \neq 0
\end{array}\right\} .
$$


We wish to simplify Equation (9.d) to take $\beta(x)=0$. We consider the change of variables $T(x, y, z)=\left(x, y-\beta(x), \tilde{x}+y \beta_{x}(x)\right)$ :

$$
\begin{array}{rlr}
T_{*} \partial_{x}=\left(\begin{array}{rrr}
1, & -\beta_{x}(x), & y \beta_{x x}(x)
\end{array}\right), \\
T_{*} \partial_{y}=\left(\begin{array}{rrr}
0, & 1, & \beta_{x}(x)
\end{array}\right), \\
T_{*} \partial_{\tilde{x}}=\left(\begin{array}{rrr}
0, & 0, & 1
\end{array}\right) .
\end{array}
$$

We compute:

$$
\begin{aligned}
& g_{f}\left(T_{*} \partial_{x}, T_{*} \partial_{x}\right)=-2 f(x, y-\beta(x))+\beta_{x}^{2}(x)+2 y \beta_{x x}(x), \\
& g_{f}\left(T_{*} \partial_{x}, T_{*} \partial_{y}\right)=0, \quad g_{f}\left(T_{*} \partial_{x}, T_{*} \partial_{\tilde{x}}\right)=1, \\
& g_{f}\left(T_{*} \partial_{y}, T_{*} \partial_{y}\right)=1, \quad g_{f}\left(T_{*} \partial_{y}, T_{*} \partial_{\tilde{x}}\right)=g_{f}\left(T_{*} \partial_{\tilde{x}}, T_{*} \partial_{\tilde{x}}\right)=0 .
\end{aligned}
$$

Thus $T^{*} g_{f}=g_{\tilde{f}}$ where

$$
\tilde{f}(x, y)=f(x, y-\beta(x))-\frac{1}{2}\left\{\beta_{x}^{2}(x)+2 y \beta_{x x}(x)\right\} .
$$

Consequently, $\tilde{f}_{y y}(x, y)=f_{y y}(x, y-\beta(x))$. Thus we may assume henceforth that $\beta(x)=0$ in Equation (9.d), i.e.

$$
f_{y y}=\left\{\begin{array}{ll}
e^{\alpha(x) y} & \text { if } c_{11}=1 \text { for } \alpha(x) \neq 0 \\
\alpha(x) y^{c} & \text { if } c_{11} \neq 1 \text { for } \alpha(x) \neq 0 \text { and } c \neq 0
\end{array}\right\}
$$

We examine these two cases seriatim. We shall use the relations:

$$
\begin{aligned}
\lambda & =f_{y y y} f_{y y}^{-1}, \quad a_{12}=-f_{x y y} f_{y y y}^{-1}, \quad \lambda^{2} a_{11}^{-2}=f_{y y}, \\
\lambda^{4} c_{122112} & =\nabla^{2} R\left(\xi_{1}, \xi_{2}, \xi_{2}, \xi_{1} ; \xi_{1}, \xi_{2}\right)=a_{11}^{3}\left\{f_{x y y y}+a_{12} f_{y y y y}\right\}, \\
\lambda^{4} c_{122111} & =\nabla^{2} R\left(\xi_{1}, \xi_{2}, \xi_{2}, \xi_{1} ; \xi_{1}, \xi_{1}\right) \\
& =a_{11}^{4}\left\{f_{x x y y}+2 a_{12} f_{x y y y}+a_{12}^{2} f_{y y y y}-f_{y} f_{y y y}\right\} .
\end{aligned}
$$

Case I. Suppose $f_{y y}=e^{\alpha(x) y}$. Then Equation (9.e) implies:

$\lambda=f_{y y y} f_{y y}^{-1}=\alpha(x), a_{12}=-f_{x y y} f_{y y y}^{-1}=-y \alpha_{x}(x) \alpha(x)^{-1}, \lambda^{2} a_{11}^{-2}=f_{y y}=e^{\alpha(x) y}$.

We use Equation (9.f) to see that:

$$
\begin{aligned}
& f_{x y y y}+a_{12} f_{\text {yyyy }}=\partial_{x}\left\{\alpha(x) e^{\alpha(x) y}\right\}-\alpha_{x}(x) \alpha(x) e^{\alpha(x) y} \\
= & \alpha_{x}(x) \cdot e^{\alpha(x) y}=a_{11}^{-3} \lambda^{4} c_{12}=\alpha(x) e^{\frac{3}{2} \alpha(x) y} c_{122112} .
\end{aligned}
$$

It now follows that $\alpha_{x}(x)=0$ so $\alpha(x)=a$ is constant. Thus we may express:

$$
f(x, y)=a^{-2} e^{a y}+u(x) y+v(x) .
$$

We then use Equation (9.e) to see

$$
\lambda=a, \quad a_{12}=0, \quad \lambda^{2} a_{11}^{-2}=e^{a y} .
$$

Equation (9.g) then leads to the identity:

$$
e^{2 a y} c_{122111}=a_{11}^{-4} \lambda^{4} c_{122111}=-f_{y} f_{y y y}=-e^{2 a y}-u(x) a e^{a y} .
$$

This implies that $u(x)=0$ and hence $f=a^{-2} e^{a y}+v(x)$. Let $w_{x}=v(x)$ and set:

$$
\begin{aligned}
T(x, y, \tilde{x}) & =\left(\begin{array}{llr}
x, & y, & \tilde{x}+2 w(x)
\end{array}\right), \\
T_{*} \partial_{x} & =\left(\begin{array}{llr}
1, & 0, & 2 v(x)
\end{array}\right), \\
T_{*} \partial_{y} & =\left(\begin{array}{lll}
0, & 1, & 0
\end{array}\right), \\
T_{*} \partial_{\tilde{x}} & =\left(\begin{array}{lll}
0, & 0, & 1
\end{array}\right)
\end{aligned}
$$

Let $\Theta:=(x, y, \tilde{x})$ and let $\tilde{\Theta}:=T \Theta$. Under this change of variables:

$$
\begin{aligned}
& g\left(T_{*} \partial_{x}, T_{*} \partial_{x}\right)(\tilde{\Theta})=-2 a^{-2} e^{a y}-2 v(x)+2 v(x)=-2 a^{-2} e^{a y}, \\
& g\left(T_{*} \partial_{x}, T_{*} \partial_{y}\right)(\tilde{\Theta})=g\left(T_{*} \partial_{y}, T_{*} \partial_{\tilde{x}}\right)(\tilde{\Theta})=g\left(T_{*} \partial_{\tilde{x}}, T_{*} \partial_{\tilde{x}}\right)(\tilde{\Theta})=0, \\
& g\left(T_{*} \partial_{x}, T_{*} \partial_{\tilde{x}}\right)(\tilde{\Theta})=g\left(T_{*} \partial_{y}, T_{*} \partial_{y}\right)(\tilde{\Theta})=1 .
\end{aligned}
$$


Thus we may take $f=a^{-2} e^{a y}$. Replacing $y$ by $y+y_{0}$ for suitably chosen $y_{0}$, then replaces $f$ by $e^{a y}$ as desired.

We now show $\mathcal{M}_{e^{a y}}$ is a homogeneous space. Clear the previous notation and set:

$$
T(x, y, \tilde{x})=\left( \pm e^{-a y_{0} / 2} x+x_{0}, y+y_{0}, \pm e^{a y_{0} / 2} \tilde{x}+\tilde{x}_{0}\right) .
$$

Then

$$
T_{*} \partial_{x}= \pm e^{-a y_{0} / 2} \partial_{x}, \quad T_{*} \partial_{y}=\partial_{y}, \quad T_{*} \partial_{\tilde{x}}=\mp e^{a y_{0} / 2} \partial_{\tilde{x}} .
$$

Set $\Theta:=(x, y, \tilde{x})$ and $\tilde{\Theta}:=T \Theta$. We show that $T$ is an isometry by verifying:

$$
\begin{aligned}
& g\left(T_{*} \partial_{x}, T_{*} \partial_{x}\right)(\tilde{\Theta})=-2 e^{-a y_{0}} e^{a\left(y+y_{0}\right)}=g\left(\partial_{x}, \partial_{x}\right)(P), \\
& g\left(T_{*} \partial_{x}, T_{*} \partial_{y}\right)(\tilde{\Theta})=0, \quad g\left(T_{*} \partial_{x}, T_{*} \partial_{\tilde{x}}\right)(\tilde{\Theta})=1, \quad g\left(T_{*} \partial_{y}, T_{*} \partial_{y}\right)(\tilde{\Theta})=1, \\
& g\left(T_{*} \partial_{y}, T_{*} \partial_{\tilde{x}}\right)(\tilde{\Theta})=0, \quad g\left(T_{*} \partial_{\tilde{x}}, T_{*} \partial_{\tilde{x}}\right)(\tilde{\Theta})=0 .
\end{aligned}
$$

Since $\left(x_{0}, y_{0}, \tilde{x}_{0}\right)$ are arbitrary, $\mathcal{I}\left(\mathcal{M}_{e^{a x}}\right)$ acts transitively on $\mathbb{R}^{3}$ so this manifold is globally homogeneous. This verifies Theorem 1.16 (3a).

Case II. Suppose that $f_{y y}=\alpha(x) y^{c}$ for $\alpha(x)>0$ and $c \neq 0$. Equation (9.e) yields

$$
\lambda=f_{y y y} f_{y y}^{-1}=c y^{-1}, \quad a_{12}=-f_{x y y} f_{y y y}^{-1}=-\frac{a_{x}(x) y}{c \alpha(x)}, \quad \lambda^{2} a_{11}^{-2}=f_{y y}=\alpha(x) y^{c} .
$$

We apply Equation (9.f) to see:

$$
\begin{aligned}
& f_{x y y y}+a_{12} f_{\text {yyyy }}=\alpha_{x}(x) c y^{c-1}-\frac{\alpha_{x}(x) y}{c \alpha(x)} c(c-1) \alpha(x) y^{c-2}=\alpha_{x}(x) y^{c-1} \\
= & a_{11}^{-3} \lambda^{4} c_{122112}=\alpha(x)^{3 / 2} y^{3 / 2 c} c y^{-1} c_{122112} .
\end{aligned}
$$

This implies

$$
\alpha_{x}(x) \alpha(x)^{-3 / 2}=c \cdot c_{122112} \cdot y^{c / 2} .
$$

Consequently $\alpha_{x}(x)=0$ so $\alpha(x)=a$ is constant. Consequently, $f_{y y}=a y^{c}$ for $c \neq 0$ and $a \neq 0$. Let $P(t)$ solve the equation $P^{\prime \prime}(t)=t^{c}$. We then have

$$
f(x, y)=a P(y)+u(x) y+v(x) .
$$

We apply Equation (9.g) with $a_{12}=0$ :

$$
\begin{aligned}
& a_{11}^{-4} \nabla^{2} R\left(\xi_{1}, \xi_{2}, \xi_{2}, \xi_{1} ; \xi_{1}, \xi_{1}\right)=-f_{y} f_{y y y}=-a P^{\prime}(y) a c y^{c-1}-u(x) a c y^{c-1} \\
= & c_{122111} \lambda^{4} a_{11}^{-4}=c_{122111} a^{2} y^{2 c} .
\end{aligned}
$$

If $c=-1$, then $P^{\prime}(y)=\ln (y)$ and this relation is impossible. Consequently $c \neq-1$ and we may conclude that $u(x)=0$. We therefore have $f=a P(y)+v(x)$. As in Case I, the constant term is eliminated and $a$ is set to 1 by making a change of variables

$$
T(x, y, \tilde{x})=\left(a^{-1 / 2} x, y, a^{1 / 2} \tilde{x}+2 w(x)\right)
$$

where $w_{x}(x)=v(x)$. Thus $f= \pm \ln (y)$ or $f= \pm y^{\varepsilon}$ for $\varepsilon \neq 0,1,2$.

Case II-a. Let $f(y)=\ln (y)$; the case $f(y)=-\ln (y)$ is similar. We know by Theorem 1.15 that $\mathcal{M}_{f}$ is not 2-curvature homogeneous and hence is not homogeneous. We clear the previous notation. For $\lambda>0$ and $\left(x_{0}, \tilde{x}_{0}\right)$ arbitrary, set:

$$
T(x, y, \tilde{x}):=\left(\lambda x+x_{0}, \lambda y, \lambda \tilde{x}+\tilde{x}_{0}+(\lambda \ln \lambda) x\right) .
$$

Let $\Theta:==(x, y, \tilde{x})$ and $\tilde{\Theta}=T \Theta$. We compute:

$$
\begin{aligned}
& T_{*} \partial_{x}=\lambda \partial_{x}+\lambda \ln \lambda \partial_{\tilde{x}}, \quad T_{*} \partial_{y}=\lambda \partial_{y}, \quad T_{*} \partial_{\tilde{x}}=\lambda \partial_{\tilde{x}}, \\
& g\left(T_{*} \partial_{x}, T_{*} \partial_{x}\right)(\tilde{\Theta})=\lambda^{2}\{-2 \ln (y)-2 \ln \lambda\}+2 \lambda^{2} \ln \lambda=\lambda^{2} g\left(\partial_{x}, \partial_{x}\right)(P), \\
& g\left(T_{*} \partial_{x}, T_{*} \partial_{y}\right)(\tilde{\Theta})=0, \quad g\left(T_{*} \partial_{x}, T_{*} \partial_{\tilde{x}}\right)(\tilde{\Theta})=\lambda^{2}, \quad g\left(T_{*} \partial_{y}, T_{*} \partial_{y}\right)(\tilde{\Theta})=\lambda^{2}, \\
& g\left(T_{*} \partial_{y}, T_{*} \partial_{\tilde{x}}\right)(\tilde{\Theta})=0, \quad g\left(T_{*} \partial_{\tilde{x}}, T_{*} \partial_{\tilde{x}}\right)=0 .
\end{aligned}
$$


This defines a transitive action by homotheties on $\mathbb{R} \times \mathbb{R}^{+} \times \mathbb{R}$, which shows that $\mathcal{M}_{\ln (y)}$ is homothety homogeneous. Moreover $T$ acts by isometries on each level set of the projection $(x, y, \tilde{x}) \mapsto y$, showing that $\mathcal{M}_{\ln (y)}$ is of cohomogeneity one.

Case II-b. Let $f(y)=y^{c}$ for $c \neq 0,1,2$. Again, Theorem 1.15 implies $\mathcal{M}_{f}$ is not 2-curvature homogeneous and hence not homogeneous. Let

$$
T(x, y, \tilde{x})=\left(\lambda^{(2-c) / 2} x+x_{0}, \lambda y, \mp \lambda^{2+(c-2) / 2} \tilde{x}+\tilde{x}_{0}\right) .
$$

We compute:

$$
\begin{aligned}
& T_{*} \partial_{x}=\lambda^{(2-c) / 2} \partial_{x}, \quad T_{*} \partial_{y}=\lambda \partial_{y}, \quad T_{*} \partial_{\tilde{x}}=\lambda^{2+(c-2) / 2} \partial_{\tilde{x}}, \\
& g\left(T_{*} \partial_{x}, T_{*} \partial_{x}\right)(T(x, y, \tilde{x}))=-2 \lambda^{(2-c)} \lambda^{c} y^{c}=\lambda^{2} g\left(\partial_{x}, \partial_{x}\right)(x, y, \tilde{x}), \\
& g\left(T_{*} \partial_{x}, T_{*} \partial_{y}\right)=0, \quad g\left(T_{*} \partial_{x}, T_{*} \partial_{\tilde{x}}\right)=\lambda^{2}, \quad g\left(T_{*} \partial_{y}, T_{*} \partial_{y}\right)=\lambda^{2}, \\
& g\left(T_{*} \partial_{y}, T_{*} \partial_{\tilde{x}}\right)=0, \quad g\left(T_{*} \partial_{\tilde{x}}, T_{*} \partial_{\tilde{x})}=0 .\right.
\end{aligned}
$$

Thus $T$ is a homothety; since $\lambda>0$ is arbitrary and since $\left(x_{0}, \tilde{x}_{0}\right)$ are arbitrary, the group of homotheties acts transitively on $M$. This verifies Theorem 1.16 (3c) and completes the proof of Theorem 1.16.

\section{The Proof of TheOrem 1.18}

We consider the family of Lorentzian manifolds given in Section 9 and consider the family of models where $\left\langle\xi_{1}, \xi_{3}\right\rangle=\left\langle\xi_{2}, \xi_{2}\right\rangle=1$ defines the inner product on the vector space $V=\operatorname{Span}\left\{\xi_{1}, \xi_{2}, \xi_{3}\right\}$. Choose the isometry between $T_{P} M$ and $(V,\langle\cdot, \cdot\rangle)$ to be defined by:

$$
\xi_{1}=a_{11}\left(\partial_{x}+f \partial_{\tilde{x}}+a_{12} \partial_{y}+a_{13} \partial_{\tilde{x}}\right), \quad \xi_{2}=\partial_{y}+a_{23} \partial_{\tilde{x}}, \quad \xi_{3}=a_{11}^{-1} \partial_{\tilde{x}}
$$

To ensure the map in question is an isometry, we require $a_{12}^{2}+2 a_{13}=0$ and $a_{12}+a_{23}=0$. This normalizes the parameters $a_{13}$ and $a_{23} ; a_{11}$ and $a_{12}$ and $\lambda$ are the free parameters of the theory.

We take $f(x, y)=\frac{1}{2} \alpha(x) y^{2}$. The only (possibly) non-zero covariant derivatives are given by:

$$
\nabla^{\ell} R\left(\partial_{x}, \partial_{y}, \partial_{y}, \partial_{x} ; \partial_{x}, \ldots, \partial_{x}\right)=\alpha^{(\ell)}(x) .
$$

The parameter $a_{12}$ plays no role in the theory and we have

$$
\nabla^{\ell} R\left(\xi_{1}, \xi_{2}, \xi_{2}, \xi_{1} ; \xi_{1}, \ldots, \xi_{1}\right)=a_{11}^{2+\ell} \alpha^{(\ell)}
$$

Suppose $k$ is given. Let $\alpha^{(k)}:=e^{-x^{2}}$ and recursively define

$$
\alpha^{(\ell)}(x):=\int_{t=-\infty}^{x} \alpha^{(\ell+1)}(t) d t \text { for } 0 \leq \ell \leq k-1 .
$$

For $x \leq-1,-x^{2} \leq x$ and consequently $\alpha^{(k)} \leq e^{x}$. Inductively, suppose $\alpha^{(\ell)} \leq e^{x}$ for $x \leq-1$. Then integration yields $\alpha^{(\ell-1)} \leq e^{x}$ for $x \leq-1$ as well. Thus the integrals in Equation (10.a) converge and $\alpha$ is a smooth function. We have $\alpha^{(\ell)}>0$ for $0 \leq \ell \leq k$; setting the functions $a_{11}^{\ell}=\left\{\alpha^{(\ell)}\right\}^{-1 /(2+\ell)}$ then yields

$$
\nabla^{\ell} R\left(\xi_{1}, \xi_{2}, \xi_{2}, \xi_{1} ; \xi_{1}, \ldots, \xi_{1}\right)=1
$$

and shows $\mathcal{M}_{\frac{1}{2} \alpha(x) y^{2}}$ is variable $k$-curvature homogeneous. Since, however, $\alpha^{(k+1)}$ vanishes when $x=0$ and is non-zero when $x \neq 0, \mathcal{M}_{\frac{1}{2} \alpha(x) y^{2}}$ is not variable $k+1$-curvature homogeneous on any neighborhood of 0 . This establishes Theorem 1.18 (1).

We take $\alpha(x)=e^{x}$. Then $\alpha^{(k)}(x)>0$ for all $x$ and for all $k$. Thus the argument given above shows $\mathcal{M}_{\frac{1}{2} e^{x} y^{2}}$ is variable $k$-curvature homogeneous for all $k$. On the other hand, by Theorem $1.16(2), \mathcal{M}_{\frac{1}{2} e^{x} y^{2}}$ is not homothety 1-curvature homogeneous. This establishes Theorem $1.18(2)$.

We consider cases to establish Theorem $1.18(3)$ : 
(1) We use Theorem 1.15(1b) and Theorem 1.16 (1) to see that the implication $(1 b) \Rightarrow(1 a)$ fails. This also shows the implication $(2 b) \Rightarrow(1 a)$ fails.

(2) If we take $f=\frac{1}{2} e^{x} y^{2}$, then this is variable $k$-curvature homogeneous for all $k$ by Theorem 1.18 (2) but not, by Theorem 1.16 (2) homothety 1-curvature homogeneous. Consequently the implications $(2 a) \Rightarrow(1 a),(2 a) \Rightarrow(1 b)$, $(2 b) \Rightarrow(1 a)$, and $(2 b) \Rightarrow(1 b)$ fail.

(3) Let $\left(S^{2}, g_{2}\right)$ denote the standard round sphere in $\mathbb{R}^{3}$. Let

$$
\mathcal{M}_{t}:=\left(M, g_{t}\right):=\left(\mathbb{R} \times S^{2}, e^{t x}\left(d x^{2}+g_{2}\right)\right)
$$

where $t \in(0, \epsilon)$ is a small positive real parameter. We showed previously that this is homothety homogeneous and hence satisfies (1b) for all $k$. We also showed it was not 0-curvature homogeneous for generic values of $t$. Note that variable 0-curvature and 0-curvature homogeneous are the same. Thus the implications $(1 b) \Rightarrow(2 a)$ and $(2 b) \Rightarrow(2 a)$ fail.

\section{ACKNOWLEDGMENTS}

Research of all the authors was partially supported by programs with FEDER funds of Xunta de Galicia (Grant GRC2013-045). Research of P. Gilkey and S. Nikčević was also partially supported by project 174012 (Serbia).

\section{REFERENCES}

[1] A. Alcolado, A. MacDougall, A. Coley, and S. Hervik, "4D neutral signature VSI and CSI spaces", J. Geom. Phys. 62 (2012), 594-603.

[2] D. Alekseevski, "Selfsimilar lorentzian manifolds", Ann. Global Anal. Geom. 3 (1985), 5984.

[3] A. Coley, R. Milson, V. Pravda, and A. Pravdová, "Vanishing scalar invariant spacetimes in higher dimensions", Classical Quantum Gravity 21 (2004), 5519-5542.

[4] A. Coley, S. Hervik, and N. Pelavas, "Lorentzian spacetimes with constant curvature invariants in three dimensions", Classical Quantum Gravity 25 (2008), 025008, 14 pp.

[5] A. Coley, S. Hervik, and N. Pelavas, "Lorentzian manifolds and scalar curvature invariants", Classical Quantum Gravity 27 (2010), 102001, 9 pp.

[6] S. Console, and C. Olmos, "Level sets of scalar Weyl invariants and cohomogeneity", Trans. Amer. Math. Soc. 360 (2008), 629-641.

[7] C. Dunn, and C. McDonald, "Singer invariants and various types of curvature homogeneity", Ann Glob. Anal. Geom. DOI 10.1007/s10455-013-9403-z.

[8] E. García-Río, P. Gilkey, and S. Nikčević, "Homogeneity of Lorentzian three-manifolds with recurrent curvature", Math. Nachr. 287 (2014), 32-47.

[9] P. Gilkey, "The Geometry of curvature homogeneous pseudo-Riemannian manifolds", Imperial College Press (2007).

[10] O. Kowalski, and A. Vanžurová, "On curvature-homogeneous spaces of type (1,3)", Math. Nachr. 284 (2011), 2127-2132.

[11] O. Kowalski, and A. Vanžurová, "On a generalization of curvature homogeneous spaces", Results. Math. 63 (2013), 129-134.

[12] W. Kühnel, and H.-B. Rademacher, "Conformal geometry of gravitational plane waves", Geometriae Dedicata 109 (2004), 175-188.

[13] B. Opozda, "Affine Versions of Singer's Theorem on Locally Homogeneous Spaces", Ann. Global Anal. Geom. 15 (1997), 187-199.

[14] F. Podesta, and A. Spiro, "Introduzione ai Gruppi di Trasformazioni", Volume of the Preprint Series of the Mathematics Department V. Volterra of the University of Ancona, Via delle Brecce Bianche, Ancona, ITALY (1996).

[15] I. Singer, "Infinitesimally homogeneous spaces", Commun. Pure Appl. Math. 13 (1960), $685-697$.

[16] M. Steller, "Conformal vector fields on spacetimes", Ann. Global Anal. Geom. 29 (2006), $293-317$.

[17] Y. Tashiro, "Complete Riemannian manifolds and some vector fields", Trans. Amer. Math. Soc. 117 (1965), 251-275.

EG: Faculty of Mathematics, University of Santiago de Compostela, Spain

E-mail address: eduardo.garcia.rio@usc.es 
PG: Mathematics Department, University of Oregon, Eugene OR 97403 USA E-mail address: gilkey@uoregon.edu

SN: Mathematical Institute, Sanu, Knez Mihailova 36, P.P. 367, 11001 Belgrade, SERBIA

E-mail address: stanan@mi.sanu.ac.rs 